\title{
ABSTRACT STOCHASTIC INTEGRODIFFERENTIAL DELAY EQUATIONS DRIVEN BY FRACTIONAL BROWNIAN MOTION
}

\author{
Mark A. McKibben and Micah Webster \\ Department of Mathematics \\ West Chester University \\ 25 University Avenue \\ West Chester, PA, 19383, U. S. A. \\ e-mail: mmckibben@wcupa.edu \\ Department of Mathematics and Computer Science \\ Goucher College \\ 1021 Dulaney Valley Road \\ Baltimore, MD, 21286, U. S. A. \\ e-mail: micah.webster@goucher.edu
}

\begin{abstract}
We investigate a class of abstract delay stochastic integrodifferential delay equations driven by a fractional Brownian motion (fBm) dependent upon a family of probability measures in a separable Hilbert space. We establish the existence and uniqueness of a mild solution and various convergence and approximation results. Finally, the analysis of two examples with numerical experiments is presented to provide numerical evidence of weak convergence.
\end{abstract}

Received: December 16, 2014; Accepted: February 17, 2015

2010 Mathematics Subject Classification: 60H05, 60H15, 60H20, 35R09.

Keywords and phrases: stochastic integrodifferential equation, fractional Brownian motion, delay equation.

The second author was supported by a Goucher College Summer Research Grant.

Communicated by K. K. Azad 


\section{Introduction}

In this paper, we will initiate an investigation of a class of abstract delay integrodifferential stochastic evolution equations driven by fractional Brownian motion ( $\mathrm{fBm}$ ) of the general form

$$
\left\{\begin{aligned}
d x(t)= & {\left[A x(t)+f_{1}\left(t, x_{t}, \mu(t)\right)+\int_{0}^{t} \mathcal{K}(t, s) f_{2}\left(s, x_{s}, \mu(s)\right) d s\right] d t } \\
& +g(t) d B^{\mathcal{H}}(t), \quad 0 \leq t \leq T \\
x(t)= & \phi(t), \quad-r \leq t \leq 0 \\
\mu(t)= & \text { probability distribution of } x(t)
\end{aligned}\right.
$$

in a real separable Hilbert space $H$. (By the probability distribution of $x(t)$, we mean $\mu(t)(\mathcal{A})=P(\{\omega \in \Omega: x(t, \omega) \in \mathcal{A}\})$, for each $\mathcal{A} \in \mathfrak{B}(H)$, where $\mathfrak{B}(H)$ stands for the Borel class on $H$.) Here, $A$ is a linear (possibly unbounded) operator which generates a strongly continuous semigroup $\{S(t): t \geq 0\}$ on $H ; \mathcal{K}(t, s)$ is a bounded linear operator on $H ; f_{i}:[0, T]$ $\times C_{r} \times \wp_{\lambda^{2}}(H) \rightarrow H(i=1,2)\left(\wp_{\lambda^{2}}(H)\right.$ denotes a particular subset of probability measures on $H)$ are given mappings; $g:[0, T] \rightarrow \mathfrak{L}(K, H)$ is a bounded, strongly measurable mapping (where $K$ is a real separable Hilbert space and $\mathfrak{L}(K, H)$ denotes the space of Hilbert-Schmidt operators from $K$ into $H$ with norm $\left.\|\cdot\|_{\mathfrak{L}(K, H)}\right), \quad\left\{B^{\mathcal{H}}(t): t \geq 0\right\}$ is a $K$-valued fBm with Hurst parameter $\mathcal{H} \in\left(\frac{1}{2}, 1\right)$; and $\phi(t) \in L^{2}\left(\Omega ; C_{r}\right)$ is an $\mathfrak{F}_{0}$-measurable random variable independent of $B^{\mathcal{H}}$ with almost sure continuous paths. (See Section 2 for notation and spaces.)

Stochastic partial functional differential equations with finite delay arise naturally in the mathematical modeling of phenomena in the natural sciences (see $[23,33,36])$ and have received a significant amount of attention. It has been shown that some applications, such as communication networks and certain financial models, exhibit a self-similarity property in the sense that 
the processes $\{x(\alpha t): 0 \leq t \leq T\}$ and $\left\{\alpha^{\mathcal{H}} x(t): 0 \leq t \leq T\right\}$ have the same law (see $[4,6]$ ). Indeed, while the case when $\mathcal{H}=1 / 2$ generates a standard Brownian motion, concrete data from a variety of applications have exhibited other values of $\mathcal{H}$, and it seems that this difference enters in a non-negligible way in the mathematical modeling of this phenomena. In fact, since $B^{\mathcal{H}}(t)$ is not a semimartingale unless $\mathcal{H}=1 / 2$, the standard stochastic calculus involving the Itó integral cannot be used in the analysis of related stochastic evolution equations. There are numerous papers devoted to the formulation of stochastic calculus for $\mathrm{fBm}[13,24,28]$ and differential/evolution equations driven by $\mathrm{fBm}[2,14,16,26]$. We provide an outline of only the necessary concomitant technical details concerning the construction of the stochastic integral driven by an $\mathrm{fBm}$ in Section 2.

Often, a more accurate model of such phenomena can be formulated by allowing the nonlinear perturbations to depend on the probability law of the state process at time $t$. A prototypical example in the finite-dimensional setting would be an interacting $M$-particle system in which (1.1) describes the dynamics of the particles $x_{1}, \ldots, x_{M}$ moving in the space $H$ in which the probability measure $\mu(t)$ is taken to be the empirical measure $\mu_{M}(t)=$ $(1 / M) \sum_{k=1}^{M} \delta_{x_{k}(t)}$, where $\delta_{x_{k}(t)}$ denotes the Dirac measure. Researchers have investigated related models concerning diffusion processes in the finite-dimensional case $[11,12,27]$. The infinite dimensional version of such models in a Hilbert space setting has only recently been examined $[1,20]$. The motivation of the present work lies primarily in formulating an extension of the work in $[1,8,15,19]$ to a more general class of abstract integrodifferential stochastic evolution equations with finite delay, now driven by fBm. Since dynamical systems with memory can lead to such random integrodifferential equations (cf. $[4,9,18,25]$ ), the present investigation is warranted.

The following is the outline of the paper. First, we make precise the necessary notation and function spaces, and gather certain preliminary results 
in Section 2. We then formulate the main results concerning the existence and uniqueness of mild solutions to (1.1) in Section 3. Next, considering the Yosida approximations of (1.1), two convergence results are established in Section 4, while Section 5 is devoted to a discussion of two concrete examples (a generalized stochastic heat equation and a Sobolev-type stochastic equation). Lastly, in Section 6, we propose a numerical scheme to approximate solutions to a special case of (1.1) and illustrate approximate solutions to the examples described in Section 5.

\section{Preliminaries}

For details on fBm, stochastic analysis, and abstract differential equations, we refer the reader to $[2,3,5,10,22,30]$ and the references therein. Throughout this paper, $K$ is a real separable Hilbert space with norm $\|\cdot\|_{K}$ and inner product $\langle\cdot, \cdot\rangle_{K}$ equipped with a complete orthonormal basis $\left\{e_{j} \mid j=1,2, \ldots\right\}$. Also, $(\Omega, \mathfrak{F}, P)$ is a complete probability space. For brevity, we suppress the dependence of all random variables on $\omega$ throughout the manuscript.

We begin by making precise the definition of a $K$-valued $\mathrm{fBm}$ and related stochastic integral used in this manuscript. The approach we use coincides with the one formulated and analyzed in $[2,24]$. Let $\left\{B_{j}^{\mathcal{H}}(t) \mid t \geq 0\right\}_{j=1}^{\infty}$ be a sequence of independent, one-dimensional $\mathrm{fBms}$ with Hurst parameter $\mathcal{H} \in(1 / 2,1)$ such that for all $j=1,2, \ldots$, the following hold:

(1) $B_{j}^{\mathcal{H}}(0)=0$,

(2) $E\left[B_{j}^{\mathcal{H}}(t)-B_{j}^{\mathcal{H}}(s)\right]^{2}=|t-s|^{2 \mathcal{H}} v_{j}$,

(3) $E\left[B_{j}^{\mathcal{H}}(1)\right]^{2}=v_{j}>0$,

(4) $\sum_{j=1}^{\infty} v_{j}<\infty$. 
In such as case, $\sum_{j=1}^{\infty} E\left\|B_{j}^{\mathcal{H}}(t) e_{j}\right\|_{K}^{2}=t^{2 \mathcal{H}} \sum_{j=1}^{\infty} v_{j}<\infty$, so that the following definition is meaningful.

Definition 2.1. For every $t \geq 0, B^{\mathcal{H}}(t)=\sum_{j=1}^{\infty} B_{j}^{\mathcal{H}}(t) e_{j}$ is a $K$-valued $\mathrm{fBm}$, where the convergence is understood in the mean-square sense.

It has been shown in $[2,16]$ that the covariance operator of $\left\{B^{\mathcal{H}}(t)\right.$ : $t \geq 0\}$ is a positive nuclear operator $Q$ such that

$$
\operatorname{tr} Q(t, s)=\frac{1}{2} \sum_{j=1}^{\infty} v_{j}\left[t^{2 \mathcal{H}}+s^{2 \mathcal{H}}-|t-s|^{2 \mathcal{H}}\right]
$$

Next, we outline the discussion leading to the definition of the stochastic integral associated with $\left\{B^{\mathcal{H}}(t): t \geq 0\right\}$ for bounded, strongly measurable functions, as presented in $[2,16]$. To begin, assume that $g:[0, T] \rightarrow \mathfrak{L}(K, H)$ is a simple function, that is, there exists $\left\{g_{i}: i=1, \ldots, n\right\} \subset \mathbb{R}$ such that

$$
g(t)=g_{i}, \quad \forall t_{i-1} \leq t \leq t_{i}
$$

where $0=t_{0}<t_{1}<\cdots<t_{n-1}<t_{n}=T$ and $\max _{1 \leq i \leq n}\left\|g_{i}\right\|_{\mathfrak{L}(K, H)}=L$.

Definition 2.2. The $H$-valued stochastic integral $\int_{0}^{T} g(t) d B^{\mathcal{H}}(t)$ is defined by

$$
\begin{aligned}
\int_{0}^{T} g(t) d B^{\mathcal{H}}(t) & =\sum_{j=1}^{\infty}\left(\int_{0}^{T} g(t) e_{j} d B_{j}^{\mathcal{H}}(t)\right) \\
& =\sum_{j=1}^{\infty}\left(\sum_{i=1}^{\infty} g_{i} e_{j}\left[B_{j}^{\mathcal{H}}\left(t_{i}\right)-B_{j}^{\mathcal{H}}\left(t_{i-1}\right)\right]\right)
\end{aligned}
$$


As argued in [2], this integral is well defined since

$$
E\left\|\int_{0}^{T} g(t) d B^{\mathcal{H}}(t)\right\|_{H}^{2} \leq L^{2} T^{2 \mathcal{H}} \sum_{j=1}^{\infty} v_{j}<\infty
$$

where the expectation, $E$, is defined by $E(y)=\int_{\Omega} y(\omega) d P$.

Since the set of simple functions is dense in the space of bounded, strongly measurable $\mathfrak{L}(K, H)$-valued functions, a standard density argument can be used to extend Definition 2.2 to the case of a general bounded, strongly measurable integrand. Furthermore, the sequence of random variables $\left\{\int_{0}^{T} g(t) e_{j} d B_{j}^{\mathcal{H}}(t): j \in \mathbb{N}\right\}$ are mutually independent, Gaussian variables. Therefore, (2.3) yields that the series in (2.2) is an $H$-valued Gaussian process. (See [14] for a more general result.)

Throughout the rest of the manuscript, $\|\cdot\|$ denotes $\|\cdot\|_{H}, \mathfrak{B} \mathfrak{L}(H)$ represents the space of all bounded linear operators on $H$, and $L^{2}(\Omega ; H)$ stands for the space of all $H$-valued random variables $y$ for which $E\|y\|^{2}$ $<\infty$.

The following spaces of measures coincide with those used in [1]; we recall them here for convenience. First, $\mathfrak{B}(H)$ stands for the Borel class on $H$ and $\wp(H)$ represents the space of all probability measures defined on $\mathfrak{B}(H)$ equipped with the weak convergence topology. Let $\lambda(x)=1+\|x\|$.

Define the space

$$
\begin{aligned}
\mathscr{C}=\{\varphi: H \rightarrow H \mid \varphi \text { is continuous and } \\
\left.\|\varphi\|_{\mathscr{C}}=\sup _{x \in H} \frac{\|\varphi(x)\|}{\lambda^{2}(x)}+\sup _{x \neq y \in H} \frac{\|\varphi(x)-\varphi(y)\|}{\|x-y\|}<\infty\right\},
\end{aligned}
$$


and for $p \geq 1$, let

$$
\begin{gathered}
\wp_{\lambda^{p}}^{s}(H)=\{m: H \rightarrow \mathbb{R} \mid m \text { is a signed measure on } H \text { such that } \\
\left.\|m\|_{\lambda^{p}}=\int_{H} \lambda^{p}(x)|m|(d x)<\infty\right\},
\end{gathered}
$$

where $|m|=m^{+}+m^{-}, \quad m=m^{+}-m^{-}$is the Jordan decomposition of $m$. Then we can define the space $\wp_{\lambda^{2}}(H)=\wp_{\lambda^{2}}^{S} \cap \wp(H)$ equipped with the metric $\rho$ given by

$$
\rho\left(v_{1}, v_{2}\right)=\sup \left\{\int_{H} \varphi(x)\left(v_{1}-v_{2}\right)(d x):\|\varphi\|_{\mathscr{C}} \leq 1\right\} .
$$

It is known that $\left(\wp_{\lambda^{2}}(H), \rho\right)$ is a complete metric space. The space of all continuous $\wp_{\lambda^{2}}(H)$-valued functions defined on $[r, T]$, denoted $\mathscr{C}_{\lambda^{2}}=$ $\mathscr{C}_{\lambda^{2}}\left([-r, T] ;\left(\wp_{\lambda^{2}}(H), \rho\right)\right)$, is complete when equipped with the metric

$$
D_{T}\left(v_{1}, v_{2}\right)=\sup _{t \in[-r, T]} \rho\left(v_{1}(t), v_{2}(t)\right), \quad \forall v_{1}, v_{2} \in \mathscr{C}_{\lambda^{2}}
$$

Next, let $r>0$. We can associate to any continuous, $\mathfrak{F}_{t}$-adapted, $H$ valued stochastic process $z(t): \Omega \rightarrow H$ another $C_{r}$-valued stochastic process $z_{t}: \Omega \rightarrow C_{r}$ by setting $z_{t}(s)=z(t+s)$, for all $t \geq 0$ and $-r \leq s \leq 0$, where we denote by $C_{r}=C([r, 0] ; H)$ the space of all continuous functions from $[r, 0]$ into $H$, equipped with the sup norm given by

$$
\|z\|_{C_{r}}=\left(\sup _{-r \leq \theta \leq 0}\left\|z_{t}(\theta)\right\|^{2}\right)^{\frac{1}{2}} .
$$

Subsequently, we can define the space

$$
X_{T, 2}=\left\{z \in C\left([-r, T] ; L^{2}(\Omega ; H)\right) \mid z \text { is } \mathfrak{F}_{t} \text {-adapted and }\|z\|_{X_{T, 2}}<\infty\right\},(2.5)
$$


which is a Banach space when equipped with the norm

$$
\|z\|_{X_{T, 2}}=\sup _{0 \leq t \leq T}\left(E\left\|z_{t}\right\|_{C_{r}}^{2}\right)^{\frac{1}{2}}
$$

In addition to the familiar Young, Hölder and Minkowski inequalities, the inequality of the form $\left(\sum_{i=1}^{n} a_{i}\right)^{m} \leq m^{n-1} \sum_{i=1}^{n} a_{i}^{m}$, where $a_{i}$ is a nonnegative constant $(i=1,2, \ldots, n)$ and $m, n \in \mathbb{N}$, will be used to establish various estimates. Finally, the following integral inequality (see [29]) plays an important role in the proofs of certain results.

Lemma 2.3. Let $u, \Psi_{1}, \Psi_{2}$ and $\Psi_{3}$ be nonnegative continuous functions defined in $\mathbb{R}^{+}$and $u_{0}$ be a nonnegative constant. If

$$
\begin{aligned}
u(t) \leq & u_{0}+\int_{0}^{t} \Psi_{1}(s) u(s) d s+\int_{0}^{t} \Psi_{1}(s)\left(\int_{0}^{s} \Psi_{2}(\tau) u(\tau) d \tau\right) d s \\
& +\int_{0}^{t} \Psi_{1}(s)\left(\int_{0}^{s} \Psi_{2}(\tau)\left(\int_{0}^{\tau} \Psi_{3}(\theta) u(\theta) d \theta\right) d \tau\right) d s
\end{aligned}
$$

for all $t \in \mathbb{R}^{+}$, then

$$
\begin{aligned}
u(t) \leq & u_{0}\left[1+\int_{0}^{t}\left\{\Psi_{1}(s) \exp \left(\int_{0}^{s} \Psi_{1}(\tau) d \tau\right)\right.\right. \\
& \left.\left.\times\left(1+\int_{0}^{s} \Psi_{2}(\tau) \exp \left(\int_{0}^{\tau}\left[\Psi_{2}(\theta)+\Psi_{3}(\theta)\right] d \theta\right) d \tau\right)\right\} d s\right]
\end{aligned}
$$

for all $t \in \mathbb{R}$.

We conclude this section with some comments regarding probability measures. The probability measure $P$ induced by an $H$-valued random variable $X$, denoted $P_{X}$, is defined by $P \circ X^{-1}: \mathfrak{B}(H) \rightarrow[0,1]$. A sequence $P_{n} \subset \wp(H)$ is said to be weakly convergent to $P$ if $\int_{\Omega} f d P_{n} \rightarrow \int_{\Omega} f d P$, for every bounded, continuous function $f: H \rightarrow \mathbb{R}$; in such case, we write 
Stochastic Integrodifferential Delay Equations Driven by fBm 765 $P_{n} \stackrel{w}{\rightarrow} P$. Next, a family $P_{n}$ is tight if for each $\varepsilon>0$, there exists a compact set $K_{\varepsilon}$ such that $P_{n}\left(K_{\varepsilon}\right) \geq 1-\varepsilon$, for all $n \in \mathbb{N}$. Bergström (see [3]) established the equivalence of tightness and relative compactness of a family of probability measures. Consequently, the Arzelà-Ascoli theorem can be used to establish tightness.

Definition 2.4. Let $P \in \wp(H)$ and $0 \leq t_{1}<t_{2}<\cdots<t_{k} \leq T$. Define $\pi_{t_{1}, t_{2}, \ldots, t_{k}}: C([0, T] ; H) \rightarrow H^{k}$ by $\pi_{t_{1}, t_{2}, \ldots, t_{k}}(X)=\left(X\left(t_{1}\right), X\left(t_{2}\right), \ldots, X\left(t_{k}\right)\right)$.

The probability measures induced by $\pi_{t_{1}, t_{2}, \ldots, t_{k}}$ are the finite-dimensional joint distributions of $P$.

Proposition 2.5 (See [22]). If a sequence $\left\{X_{n}\right\}$ of H-valued random variables converges weakly to an $H$-valued random variable X in $L^{2}(\Omega ; H)$, then the sequence of finite-dimensional joint distributions corresponding to $\left\{P_{X_{n}}\right\}$ converges weakly to the finite-dimensional joint distribution of $P_{X}$.

The next theorem, in conjunction with Proposition 2.5, is the main tool in establishing a convergence result in Section 4.

Theorem 2.6. Let $\left\{P_{n}\right\} \subset \wp(H)$. If the sequence of finite-dimensional joint distributions corresponding to $\left\{P_{n}\right\}$ converges weakly to the finitedimensional joint distribution of $P$ and $\left\{P_{n}\right\}$ is relatively compact, then $P_{n} \stackrel{w}{\rightarrow} P$.

\section{Existence and Uniqueness Results}

We begin by establishing existence and uniqueness of mild solutions to (1.1). We impose the following conditions on (1.1), which are assumed throughout the manuscript unless otherwise specified:

(A1) $A$ is the infinitesimal generator of a $C_{0}$-semigroup $\{S(t): t \geq 0\}$ on $H$ such that $\|S(t)\|_{\mathfrak{B} \mathfrak{L}(H)} \leq M \exp (\alpha t)$, for all $0 \leq t \leq T$, for some $M \geq 1$ 
and $\alpha>0$;

(A2) $f_{i}:[0, T] \times C_{r} \times \wp_{\lambda^{2}}(H) \rightarrow H(i=1,2)$ satisfies

(a) $\| f_{i}\left(t, x_{t}, \mu(t)\right) \leq \bar{M}_{f_{i}}\left[1+\left\|x_{t}\right\|_{C_{r}}+\|\mu(t)\|_{\lambda^{2}}\right]$,

(b)

$\left\|f_{i}\left(t, x_{t}, \mu(t)\right)-\right\| f_{i}\left(t, y_{t}, v(t)\right) \| \leq M_{f_{i}}\left[\left\|x_{t}-y_{t}\right\|_{C_{r}}+\rho(\mu(t), v(t))\right]$,

globally on $[0, T] \times C_{r} \times \wp_{\lambda^{2}}(H)$, for some positive constants $M_{f_{i}}$ and $\bar{M}_{f_{i}} ;$

(A3) $\{\mathcal{K}(t, s): t, s \in[0, T]\} \subset \mathfrak{B} \mathfrak{L}(H)$ are such that $\|\mathcal{K}(t, s)\|_{\mathfrak{B} \mathfrak{L}(H)} \leq$ $M_{\mathcal{K}}$, for all $t, s \in[0, T]$, for some positive constant $M_{\mathcal{K}}$;

(A4) $g:[0, T] \rightarrow \mathfrak{L}(K, H)$ is a bounded, strongly measurable mapping;

(A5) $\left\{B^{\mathcal{H}}(t): t \geq 0\right\}$ is a $K$-valued $\mathrm{fBm}$;

(A6) $\phi(t) \in L^{2}\left(\Omega ; C_{r}\right)$ is an $\mathfrak{F}_{0}$-measurable random variable independent of $B^{\mathcal{H}}$ with almost sure continuous paths.

We write $M_{S}=\max _{0 \leq t \leq T}\|S(t)\|_{\mathfrak{B} \mathfrak{L}(H)}$, which is finite by (A1). A mild solution to (1.1) is defined as follows.

Definition 3.1. A continuous stochastic process $x:[-r, T] \rightarrow H$ is a mild solution of (1.1) if

(1) $x(t)$ is $\mathfrak{F}_{t}$-adapted, for each $-r \leq t \leq T$,

(2) $\int_{0}^{T}\|x(s)\|^{2} d s<\infty$, almost surely $[P]$,

$$
x(t)=S(t) \phi(0)+\int_{0}^{t} S(t-s) f_{1}\left(s, x_{s}, \mu(s)\right) d s
$$




$$
\begin{aligned}
& +\int_{0}^{t} S(t-s) \int_{0}^{t} \mathcal{K}(s, \tau) f_{2}\left(\tau, x_{\tau}, \mu(\tau)\right) d \tau d s \\
& +\int_{0}^{t} S(t-s) g(s) d B^{\mathcal{H}}(s), 0 \leq t \leq T, \text { almost surely }[P],
\end{aligned}
$$

(4) $x(t)=\phi(t),-r \leq t \leq 0$, almost surely $[P]$.

The following technical properties involving the stochastic integral $\int_{0}^{t} S(t-s) g(s) d B^{\mathcal{H}}(s)$, under assumptions (A1), (A4) and (A5), are used in the proofs of the main results in this paper.

Lemma 3.2. Assume (A1), (A4) and (A5). Then, for all $0 \leq t \leq T$,

(i) $E\left\|\int_{0}^{t} S(t-s) g(s) d B^{\mathcal{H}}(s)\right\|^{2} \leq C_{t} \sum_{j=1}^{\infty} v_{j}$,

(ii) $\lim _{h \rightarrow 0} E\left\|\int_{0}^{t}[S(t+h-s)-S(t-s)] g(s) d B^{\mathcal{H}}(s)\right\|^{2}=0$,

where $C_{t}$ is a positive constant depending on $t,\{S(t): t \geq 0\}, L$ (cf. (2.1)), and $\left\{v_{j}: j \in \mathbb{N}\right\}$ is defined as in the discussion leading to Definition 2.1.

Proof. Property (i) can be established as in Lemma 6 in [2]. To verify property (ii), let $0 \leq t \leq T$ and observe that

$$
\begin{aligned}
& E\left\|\int_{0}^{t}[S(t+h-s)-S(t-s)] g(s) d B^{\mathcal{H}}(s)\right\|^{2} \\
= & E\left\|\sum_{j=1}^{\infty} \int_{0}^{t}[S(t+h-s)-S(t-s)] g(s) e_{j} d B_{j}^{\mathcal{H}}(s)\right\|^{2} \\
\leq & 2 E\left\|\sum_{j=1}^{m} \int_{0}^{t}[S(t+h-s)-S(t-s)] g(s) e_{j} d B_{j}^{\mathcal{H}}(s)\right\|^{2}
\end{aligned}
$$




$$
+2 E\left\|\sum_{j=m+1}^{\infty} \int_{0}^{t}[S(t+h-s)-S(t-s)] g(s) e_{j} d B_{j}^{\mathcal{H}}(s)\right\|^{2}
$$

The strong continuity of $S(\cdot)$, together with (A4), guarantees that (3.2) goes to zero as $h \rightarrow 0$. To argue (3.3) goes to zero, we must first assume $g$ is simple as defined in (2.1). Arguing as in [2] yields the estimate

$$
E\left\|\int_{0}^{t}[S(t+h-s)-S(t-s)] g(s) e_{j} d B_{j}^{\mathcal{H}}(s)\right\|^{2} \leq C_{t} t^{2 \mathcal{H}} v_{j},
$$

where $C_{t}$ is defined as in part (i) of this lemma. Using (3.4) in (3.3) yields

$$
\begin{aligned}
& E\left\|\sum_{j=m+1}^{\infty} \int_{0}^{t}[S(t+h-s)-S(t-s)] g(s) e_{j} d B_{j}^{\mathcal{H}}(s)\right\|^{2} \\
& \leq C_{t} t^{2 \mathcal{H}} \sum_{j=m+1}^{\infty} v_{j}<\infty .
\end{aligned}
$$

Therefore, (3.3) goes to zero as $m \rightarrow \infty$. As such, property (ii) holds for a simple function $g$. It is not difficult to extend the argument using density to a general bounded, strongly measurable function $g$. This completes the proof.

The first result is as follows.

Theorem 3.3. If (A1)-(A6) hold, then (1.1) has a unique mild solution $x \in X_{T, 2}$ with corresponding probability law $\mu \in \mathscr{C}_{\lambda^{2}}$, provided that $\alpha_{1}+\alpha_{2}<1$, where $\alpha_{1}, \alpha_{2}$ are positive constants independent of $T$ (see (3.18)).

Proof. Let $\mu \in \mathscr{C}_{\lambda^{2}}$ be fixed and define the solution map $\Phi: X_{T, 2} \rightarrow$ $X_{T, 2}$ by 


$$
\begin{aligned}
(\Phi x)(t)= & S(t) \phi(0)+\int_{0}^{t} S(t-s) f_{1}\left(s, x_{s}, \mu(s)\right) d s \\
& +\int_{0}^{t} S(t-s) \int_{0}^{s} \mathcal{K}(s, \tau) f_{2}\left(\tau, x_{\tau}, \mu(\tau)\right) d \tau d s \\
& +\int_{0}^{t} S(t-s) g(s) d B^{\mathcal{H}}(s) \\
= & S(t) \phi(0)+\sum_{i=1}^{3} I_{i}^{x}(t), \quad 0 \leq t \leq T, \\
(\Phi x)(t)= & \phi(t), \quad-r \leq t \leq 0 .
\end{aligned}
$$

To see that $\Phi$ is well defined, we first verify the $L^{2}(\Omega ; H)$-continuity of $\Phi$ on $[0, T]$. Let $x \in X_{T, 2}, 0<t_{1}<T$, and $|h|$ be sufficiently small (so that all terms are well defined). Observe that

$$
\begin{aligned}
& E\left\|(\Phi x)\left(t_{1}+h\right)-(\Phi x)\left(t_{1}\right)\right\|^{2} \\
\leq & 8\left[E\left\|\left(\left(S\left(t_{1}+h\right)-S\left(t_{1}\right)\right) \phi(0)\right)\right\|^{2}+\sum_{i=1}^{3} E\left\|I_{i}^{x}\left(t_{1}+h\right)-I_{i}^{x}\left(t_{1}\right)\right\|^{2}\right] .
\end{aligned}
$$

Since the semigroup property enables us to write

$$
\begin{aligned}
& E\left\|\left(\left(S\left(t_{1}+h\right)-S\left(t_{1}\right)\right) \phi(0)\right)\right\|^{2} \\
= & E\left\|\left(S(h)\left(S\left(t_{1}\right) \phi(0)\right)-S\left(t_{1}\right) \phi(0)\right)\right\|^{2},
\end{aligned}
$$

the strong continuity of $S(\cdot)$ implies that the right-hand side of (3.8) goes to 0 as $|h| \rightarrow 0$. Next, using the Hölder inequality along with (A2) yields

$$
\begin{aligned}
& E\left\|\int_{t_{1}}^{t_{1}+h} S\left(t_{1}+h-s\right) f_{1}\left(s, x_{s}, \mu(s)\right) d s\right\|^{2} \\
\leq & 4\left(\bar{M}_{f_{1}}\right)^{2} M_{s}^{2} h^{2}\left[1+\|x\|_{X_{T, 2}}^{2}+\sup _{t_{1} \leq s \leq t_{1}+h}\|\mu(s)\|_{\lambda^{2}}^{2}\right],
\end{aligned}
$$


which tends to 0 as $|h| \rightarrow 0$. Also,

$$
\begin{aligned}
& E\left\|\int_{0}^{t_{1}}[S(h)-I] S\left(t_{1}-s\right) f_{1}\left(s, x_{s}, \mu(s)\right) d s\right\|^{2} \\
\leq & T E\left(\int_{0}^{t_{1}}\left\|[S(h)-I] S\left(t_{1}-s\right) f_{1}\left(s, x_{s}, \mu(s)\right) d s\right\|^{2}\right) \\
= & T \int_{0}^{t_{1}}\left\|[S(h)-I] S\left(t_{1}-s\right)\right\|_{\mathfrak{B} \mathfrak{L}(H)}^{2} E\left\|f_{1}\left(s, x_{s}, \mu(s)\right)\right\|^{2} d s,
\end{aligned}
$$

and subsequently, using

$$
\left\|f_{1}\left(s, x_{s}, \mu(s)\right)\right\|^{2} \leq \bar{M}_{f_{1}}\left[1+\|x\|_{T, 2}+\sup _{0 \leq s \leq T}\|\mu(s)\|_{\lambda^{2}}^{2}\right]<\infty
$$

and $\|S(t)\|_{\mathfrak{B} \mathfrak{L}(H)} \leq M_{S}$, for all $t$, we can invoke dominated convergence theorem in order to use the strong continuity of $S(\cdot)$ to conclude the middle term of (3.10) goes to 0 as $|h| \rightarrow 0$. Since $E\left\|I_{1}^{x}\left(t_{1}+h\right)-I_{1}^{x}\left(t_{1}\right)\right\|^{2}$ is dominated by a sum of constant multiples of the right-sides of (3.9)-(3.10), we conclude it goes to 0 as $|h| \rightarrow 0$.

As is the argument for $E\left\|I_{1}^{x}\left(t_{1}+h\right)-I_{1}^{x}\left(t_{1}\right)\right\|^{2}$, (A2)-(A3) imply

$$
\begin{aligned}
& E\left\|\int_{t_{1}}^{t_{1}+h} S\left(t_{1}+h-s\right) \int_{0}^{s} \mathcal{K}(s, \tau) f_{1}\left(\tau, x_{\tau}, \mu(\tau)\right) d \tau d s\right\|^{2} \\
\leq & 2\left(\bar{M}_{f_{1}} M_{\mathcal{K}} M_{s} h\right)^{2}\left(3 t_{1}^{2}+3 h t_{1}+h^{2}\right) \\
& \times\left[1+\|x\|_{X_{T, 2}}^{2}+\sup _{t_{1} \leq s \leq t_{1}+h}\|\mu(s)\|_{\lambda^{2}}^{2}\right],
\end{aligned}
$$

which tends to 0 as $|h| \rightarrow 0$. Also,

$$
\begin{aligned}
& E\left\|\int_{0}^{t_{1}}[S(h)-I] S\left(t_{1}-s\right) \int_{0}^{s} \mathcal{K}(s, \tau) f_{1}\left(\tau, x_{\tau}, \mu(\tau)\right) d \tau d s\right\|^{2} \\
\leq & T E\left(\int_{0}^{t_{1}}\left\|[S(h)-I] S\left(t_{1}-s\right) \int_{0}^{s} \mathcal{K}(s, \tau) f_{1}\left(\tau, x_{\tau}, \mu(\tau)\right) d \tau d s\right\|^{2}\right)
\end{aligned}
$$




$$
\leq T \int_{0}^{t_{1}}\left\|[S(h)-I] S\left(t_{1}-s\right)\right\|_{\mathfrak{B} \mathfrak{L}(H)}^{2}\left\{\left\{\int_{0}^{s} E\left\|\mathcal{K}(s, \tau) f_{1}\left(\tau, x_{\tau}, \mu(\tau)\right)\right\|^{2} d \tau\right\} d s,\right.
$$

for which dominated convergence theorem applies by (A2)-(A3), and thus the strong continuity of $S(\cdot)$ concludes the middle term of (3.12) goes to 0 as $|h| \rightarrow 0$. Since $E\left\|I_{2}^{x}\left(t_{1}+h\right)-I_{2}^{x}\left(t_{1}\right)\right\|^{2}$ is dominated by a sum of constant multiples of the right-sides of (3.11)-(3.12), we conclude it goes to 0 as $|h| \rightarrow 0$.

It remains to show

$$
E\left\|I_{3}^{x}\left(t_{1}+h\right)-I_{3}^{x}\left(t_{1}\right)\right\|^{2} \rightarrow 0
$$

as $|h| \rightarrow 0$. Observe that

$$
\begin{aligned}
& E\left\|I_{3}^{x}\left(t_{1}+h\right)-I_{3}^{x}\left(t_{1}\right)\right\|^{2} \\
= & E\left\|\int_{0}^{t_{1}+h} S\left(t_{1}+h-s\right) g(s) d B^{\mathcal{H}}(s)-\int_{0}^{t_{1}} S\left(t_{1}-s\right) g(s) d B^{\mathcal{H}}(s)\right\|^{2} \\
= & E \| \int_{t_{1}}^{t_{1}+h} S\left(t_{1}+h-s\right) g(s) e_{j} d B_{j}^{\mathcal{H}}(s) \\
& +\int_{0}^{t_{1}}\left[S\left(t_{1}+h-s\right)-S\left(t_{1}-s\right)\right] g(s) d B^{\mathcal{H}}(s) \|^{2} \\
\leq & 2 E\left\|\sum_{j=1}^{\infty} \int_{t_{1}}^{t_{1}+h} S\left(t_{1}+h-s\right) g(s) e_{j} d B_{j}^{\mathcal{H}}(s)\right\|^{2} \\
& +2 E\left\|\int_{0}^{t_{1}}\left[S\left(t_{1}+h-s\right)-S\left(t_{1}-s\right)\right] g(s) d B^{\mathcal{H}}(s)\right\|^{2}
\end{aligned}
$$


where that

$$
\begin{aligned}
& \left\|\sum_{j=1}^{\infty} \int_{t_{1}}^{t_{1}+h} S\left(t_{1}+h-s\right) g(s) e_{j} d B_{j}^{\mathcal{H}}(s)\right\|^{2} \\
= & \left\|\sum_{j=1}^{\infty} \int_{0}^{h} S(u) g\left(t_{1}+h-u\right) e_{j} d B_{j}^{\mathcal{H}}\left(t_{1}+h-u\right)\right\|^{2} .
\end{aligned}
$$

Using the property $E\left[B_{j}^{\mathcal{H}}(s)-B_{j}^{\mathcal{H}}(t)\right]^{2}=|t-s|^{2 \mathcal{H}} v_{j}$ with $s=t_{1}+h$ and $t=t_{1}$ enables us to conclude the right-side of (3.14) goes to 0 as $|h| \rightarrow 0$. By Lemma 3.2(ii) and (3.14), the right-side of (3.13) goes to 0 as $|h| \rightarrow 0$, and therefore, we have shown $E\left\|I_{3}\left(t_{1}+h\right)-I_{3}\left(t_{1}\right)\right\|^{2} \rightarrow 0$ as $|h| \rightarrow 0$. Consequently, we can conclude from (3.7) that $\Phi$ is $L^{2}(\Omega ; H)$ continuous on $[0, T]$.

Next, to see that $\Phi\left(X_{T, 2}\right) \subset X_{T, 2}$, let $x \in X_{T, 2}$ and $t \in[0, T]$. Since $\phi \in L^{2}\left(\Omega ; C_{r}\right)$, it follows that

$$
\sup _{-r \leq \theta \leq 0}\left\{E\|(\Phi x)(t+\theta)\|^{2}:-r \leq t+\theta \leq 0\right\}<\infty .
$$

For all $r \leq \theta \leq 0$ for which $t+\theta>0$, standard computations involving the Hölder inequality, (A1)-(A5), and Lemma 3.2(i) yield the following estimates:

$$
\begin{aligned}
& E\left(\sup _{-r \leq \theta \leq 0}\|S(t+\theta) \phi(0)\|^{2}\right) \leq M_{s}^{2}\|\phi\|_{C_{r}}^{2}, \\
& E\left(\sup _{-r \leq \theta \leq 0}\left\|I_{1}^{x}(t+\theta)\right\|^{2}\right) \leq 4\left(T M_{S} M_{f_{1}}\right)^{2}\left[1+\|x\|_{X_{T, 2}}^{2}+\sup _{0 \leq \theta \leq T}\|\mu(\theta)\|_{\lambda^{2}}^{2}\right], \\
& E\left(\sup _{-r \leq \theta \leq 0}\left\|I_{2}^{x}(t+\theta)\right\|^{2}\right) \leq 4\left(T M_{S} M_{f_{2}} M_{\mathcal{K}}\right)^{2}\left[1+\|x\|_{X_{T, 2}}^{2}+\sup _{0 \leq \theta \leq T}\|\mu(\theta)\|_{\lambda^{2}}^{2}\right],
\end{aligned}
$$




$$
E\left(\sup _{-r \leq \theta \leq 0}\left\|I_{3}^{x}(t+\theta)\right\|^{2}\right) \leq\left(M_{s} L\right)^{2} T^{2 \mathcal{H}} \sum_{j=1}^{\infty} v_{j} .
$$

Using these estimates and (3.6), we conclude that

$$
\sup _{-r \leq \theta \leq 0}\left\{E\|(\Phi x)(t+\theta)\|^{2}: 0 \leq t+\theta \leq T\right\}<\infty .
$$

Thus, (3.15) and (3.16) imply that $E\left\|(\Phi x)_{t}\right\|_{C_{r}}^{2}<\infty$, for all $t \in[0, T]$, so that $\Phi x \in X_{T, 2}$. Since the $\mathfrak{F}_{t}$-measurability of $(\Phi x)(t)$ is easily verified, we conclude that $\Phi$ is well defined.

Next, we show that $\Phi$ has a unique fixed point. For any $x, y \in X_{T, 2}$, (3.6) implies that

$$
E\left\|(\Phi x)_{t}-(\Phi y)_{t}\right\|_{C_{r}}^{2} \leq 4 E\left[\sup _{-r \leq \theta \leq 0}\left(\sum_{i=1}^{3}\left\|I_{i}^{x}(t+\theta)-I_{i}^{y}(t+\theta)\right\|^{2}\right)\right] .
$$

Standard computations yield

$$
\begin{aligned}
E\left\|(\Phi x)_{t}-(\Phi y)_{t}\right\|_{C_{r}}^{2} \leq 4 T M_{s}^{2}[ & M_{f_{1}}^{2} \int_{0}^{t} E\left\|x_{\theta}-y_{\theta}\right\|_{C_{r}}^{2} d \theta+T\left(M_{f_{2}} M_{\mathcal{K}}\right)^{2} \\
& \left.\times \int_{0}^{t} \int_{0}^{\theta} E\left\|x_{\tau}-y_{\tau}\right\|_{C_{r}}^{2} d \tau d \theta\right], 0 \leq t \leq T .
\end{aligned}
$$

To prove that $\Phi^{N}$ is a strict contraction, for some $N$, we proceed in two cases. First, if $T \leq 1$, then $T^{2} \leq 1$, so that we can continue (3.17) to obtain

$$
\begin{gathered}
E\left\|(\Phi x)_{t}-(\Phi y)_{t}\right\|_{C_{r}}^{2} \\
\leq \alpha_{1} \int_{0}^{t} E\left\|x_{\theta}-y_{\theta}\right\|_{C_{r}}^{2} d \theta+\alpha_{2} \int_{0}^{t} \int_{0}^{\theta} E\left\|x_{\tau}-y_{\tau}\right\|_{C_{r}}^{2} d \tau d \theta
\end{gathered}
$$

where $\alpha_{1}=4 M_{s}^{2} M_{f_{1}}^{2}$ and $\alpha_{2}=4\left(M_{s} M_{f_{2}} M_{\mathcal{K}}\right)^{2}$ are independent of $T$. 
Inductively, it can be shown that for each $n \geq 1$, that is $\alpha_{n, j-n+1}$ is the $(j-n+1)$ th term of the quantity $\left(\alpha_{1}+\alpha_{2}\right)^{n}$, then

$$
E\left\|(\Phi x)_{t}-(\Phi y)_{t}\right\|_{C_{r}}^{2} \leq\left(\sum_{j=n}^{2 n} \alpha_{n, j-n+1} \frac{t^{j}}{j !}\right)\|x-y\|_{X_{T, 2}}^{2},
$$

and subsequently, after taking the supremum over $[0, T]$,

$$
\begin{aligned}
& \left\|(\Phi x)_{t}-(\Phi y)_{t}\right\|_{X_{T, 2}}^{2} \\
\leq & \left(\sum_{j=n}^{2 n} \alpha_{n, j-n+1} \frac{T^{j}}{j !}\right)\|x-y\|_{X_{T, 2}}^{2}=z_{n}\|x-y\|_{X_{T, 2}}^{2} .
\end{aligned}
$$

Clearly, $\alpha_{n, j-n+1} \rightarrow 0$ as $n \rightarrow \infty$, for all $j \rightarrow 1$, and $\sum_{j=n}^{2 n} \alpha_{n, j-n+1}$ $\leq 1$, for all $n \geq 1$, since $\alpha_{1}+\alpha_{2}<1$ by assumption. Since $\lim _{j \rightarrow \infty}\left(T^{j} / j\right.$ !) $=0$, for all $T$, it follows from [21, Theorem 4, p. 74] that $z_{n} \rightarrow 0$ as $n \rightarrow \infty$. Thus, there is an $N \in \mathbb{N}$ such that $z_{N}<1$, thereby showing $\Phi^{N}$ is a strict contraction (cf. (3.19)). Now suppose $T>1$, so that $T^{2}>1$ and continuing (3.17) yields

$$
\begin{aligned}
& E\left\|(\Phi x)_{t}-(\Phi y)_{t}\right\|_{C_{r}}^{2} \\
\leq & T^{2}\left[\alpha_{1} \int_{0}^{t} E\left\|x_{\theta}-y_{\theta}\right\|_{C_{r}}^{2} d \theta+\alpha_{2} \int_{0}^{t} \int_{0}^{\theta} E\left\|x_{\tau}-y_{\tau}\right\|_{C_{r}}^{2} d \tau d \theta\right], \quad 0 \leq t \leq T,
\end{aligned}
$$

so that reasoning as above leads to

$$
\left\|(\Phi x)_{t}-(\Phi y)_{t}\right\|_{X_{T, 2}}^{2} \leq z_{n} T^{2}\|x-y\|_{X_{T, 2}}^{2} .
$$

Therefore, we can deduce that there is an $N^{*} \in \mathbb{N}$ such that $z_{N^{*}}<1 / T^{2}$, again showing $\Phi^{N^{*}}$ is a strict contraction. Thus, for a given $\mu \in \mathscr{C}_{\lambda^{2}}$ and 
$T>0, \Phi$ has a unique fixed point $x_{\mu} \in X_{T, 2}$. Since $\left(\Phi x_{\mu}\right)(t)=\phi(t)$ by (3.6), for $-r \leq t \leq 0$, we conclude the $x_{\mu}$ is a mild solution of (1.1).

To complete the proof, we must show that $\mu$ is the probability law of $x_{\mu}$. Toward this end, let $\mathscr{L}\left(x_{\mu}\right)=\left\{\mathscr{L}\left(x_{\mu}(t)\right): t \in[r, T]\right\}$ represent the probability law of $x_{\mu}$ and define the map $\Psi: \mathscr{C}_{\lambda^{2}} \rightarrow \mathscr{C}_{\lambda^{2}}$ by $\Phi(\mu)=\mathscr{L}\left(x_{\mu}\right)$. It is not difficult to see that $\mathscr{L}\left(x_{\mu}(t)\right) \in \wp_{\lambda^{2}}(H)$, for all $t \in[r, T]$ since $x_{\mu} \in X_{T, 2}$ and $\phi \in L^{2}\left(\Omega ; C_{r}\right)$. Now, to verify the continuity of the map $t \rightarrow \mathscr{L}\left(x_{\mu}(t)\right)$, first let $r \leq c \leq 0$ and take $|h|>0$ small enough to ensure that $r \leq c+h$ $\leq 0$. For all $\operatorname{such} c$,

$$
E\left\|x_{\mu}(c+h)-x_{\mu}(c)\right\|^{2}=E\|\phi(c+h)-\phi(c)\|^{2} \rightarrow 0 \text { as } h \rightarrow 0
$$

by assumption. Next, for $0 \leq c \leq T$, note that for sufficiently small $|h|>0$,

$$
\begin{aligned}
& E\left\|x_{\mu}(c+h)-x_{\mu}(c)\right\|^{2} \\
\leq & 8\left[E\|(S(c+h)-S(c)) \phi(0)\|^{2}+\sum_{i=1}^{3} E\left\|I_{i}^{x_{\mu}}(c+h)-I_{i}^{x_{\mu}}(c)\right\|^{2}\right] .
\end{aligned}
$$

An argument similar to the one used to verify the continuity of $\Phi$ can be used to then deduce from (3.20)-(3.21) that

$$
\lim _{h \rightarrow 0} E\left\|x_{\mu}(c+h)-x_{\mu}(c)\right\|^{2} \rightarrow 0, \quad \forall-r \leq c \leq T .
$$

Consequently, since for all $c \in[-r, T]$ and $\varphi \in \mathscr{C}_{\lambda^{2}}$, it is the case that

$$
\begin{aligned}
& \left|\int_{H} \varphi(x)\left(\mathscr{L}\left(x_{\mu}(c+h)\right)-\mathscr{L}\left(x_{\mu}(c)\right)\right)(d x)\right| \\
= & \left|\int_{\Omega}\left[\varphi\left(x_{\mu}(c+h ; \omega)\right)-\varphi\left(x_{\mu}(c ; \omega)\right)\right] d \omega\right|
\end{aligned}
$$




$$
\begin{aligned}
& =\left|E\left[\varphi\left(x_{\mu}(c+h)\right)-\varphi\left(x_{\mu}(c)\right)\right]\right| \\
& \leq\|\varphi\|_{\mathscr{C}} E\left\|\varphi\left(x_{\mu}(c+h)\right)-\varphi\left(x_{\mu}(c)\right)\right\|,
\end{aligned}
$$

and so we conclude that

$$
\begin{aligned}
& \rho\left(\mathscr{L}\left(x_{\mu}(c+h)\right), \mathscr{L}\left(x_{\mu}(c)\right)\right) \\
= & \sup _{\|\varphi\|_{\mathscr{C}} \leq 1} \int_{H} \varphi(x)\left(\mathscr{L}\left(x_{\mu}(c+h)\right)-\mathscr{L}\left(x_{\mu}(c)\right)\right)(d x) \rightarrow 0 \text { as } h \rightarrow 0,
\end{aligned}
$$

for any $c \in[-r, T]$. Hence, $t \mapsto \mathscr{L}\left(x_{\mu}(t)\right)$ is a continuous map, so that $\mathscr{L}\left(x_{\mu}\right) \in \mathscr{C}_{\lambda^{2}}$. This shows that $\Psi$ is well-defined.

Lastly, we show that $\Psi$ has a unique fixed point in $\mathscr{C}_{\lambda^{2}}$. Let $\mu, v \in \mathscr{C}_{\lambda^{2}}$ and let $x_{\mu}, x_{v}$ be the corresponding mild solutions of (1.1). Standard computations produce

$$
\begin{aligned}
& E\left\|\left(x_{\mu}\right)_{t}-\left(x_{v}\right)_{t}\right\|^{2} \\
\leq & C_{1} \int_{0}^{t} E\left\|\left(x_{\mu}\right)_{\theta}-\left(x_{v}\right)_{\theta}\right\|_{C_{r}}^{2} d \theta \\
& +C_{2} \int_{0}^{t} \int_{0}^{\theta} E\left\|\left(x_{\mu}\right)_{t}-\left(x_{v}\right)_{t}\right\|_{C_{r}}^{2} d \tau d \theta+C_{3} D_{T}^{2}(\mu, v), \quad 0 \leq t \leq T,
\end{aligned}
$$

where

$$
\begin{aligned}
& C_{1}=16 T\left(M_{S} M_{f_{1}}\right)^{2}, \\
& C_{2}=16\left(T M_{S} M_{f_{2}} M_{K}\right)^{2}, \\
& C_{3}=C_{1} T+C_{2} T^{2} .
\end{aligned}
$$

Hence, applying Lemma 2.3 and then taking the supremum over $[0, T]$ yields 


$$
\left\|x_{\mu}-x_{v}\right\|_{X_{T, 2}}^{2} \leq \zeta_{T} D_{T}^{2}(\mu, v)
$$

where $\zeta_{T}=C_{3}\left[1+\left(C_{1}+C_{2}\right) T \exp \left(\left(C_{1}+C_{2}\right) T\right)\right](1+T \exp (T))$. We can choose $T$ small enough so that $\zeta_{T}<1$; denote such a $T$ by $T^{*}$. Since

$$
\rho\left(\mathscr{L}\left(x_{\mu}(t)\right), \mathscr{L}\left(x_{v}(t)\right)\right) \leq E\left\|x_{\mu}(t)-x_{n} u(t)\right\|, \quad \forall-r \leq t \leq T,
$$

we have

$$
\begin{aligned}
\|\Psi(\mu)-\Psi(v)\|_{\mathscr{C}_{\lambda^{2}}}^{2} & =D_{T^{*}}^{2}(\Psi(\mu)-\Psi(v)) \leq \sup _{t \in\left[-r, T^{*}\right]} E\left\|x_{\mu}(t)-x_{v}(t)\right\|^{2} \\
& =\left\|x_{\mu}-x_{v}\right\|_{X_{T, 2}}^{2}<\zeta_{T^{*}} D_{T^{*}}^{2}(\mu, v)
\end{aligned}
$$

by (3.23), so that $\Psi$ is a strict contraction on $\mathscr{C}_{\lambda^{2}}\left(\left[-r, T^{*}\right] ;\left(\wp_{\lambda^{2}}(H), \rho\right)\right)$.

Thus, (1.1) has a unique mild solution on $\left[0, T^{*}\right]$ with probability distribution

$$
\mu \in \mathscr{C}_{\lambda^{2}}\left(\left[-r, T^{*}\right] ;\left(\wp_{\lambda^{2}}(H), \rho\right)\right) .
$$

Due to continuity, this process can be repeated finitely many times to extend the solution to the entire interval $[0, T]$, thereby completing the proof.

\section{Convergence Results}

For each $n \geq 1$, consider the Yosida approximation of (1.1) given by

$$
\left\{\begin{aligned}
d x_{n}(t)= & {\left[A x_{n}(t)+n R(n ; A) f_{1}\left(t,\left(x_{n}\right)_{t} \mu(t)\right)\right.} \\
& \left.\quad+\int_{0}^{t} K(t, s) n R(n ; A) f_{2}\left(s,\left(x_{n}\right)_{s}, \mu(s)\right) d s\right] d t \\
& +n R(n ; A) g(t) d B^{\mathcal{H}}(t), \quad 0 \leq t \leq T, \\
x_{n}(t)= & n R(n ; A) \phi(t), \quad-r \leq t \leq 0, \\
\mu_{n}(t)= & \text { probability distribution of } x_{n}(t),
\end{aligned}\right.
$$

where $R(n ; A)=(I-n A)^{-1}$ is the resolvent operator of $A$. Assuming $f_{1}, f_{2}$ 
and $K$ are such that the assumptions of Theorem 3.3, one can invoke the same theorem to deduce that (4.1) has a unique mild solution $x_{n} \in X_{T, 2}$ with probability law $\mu_{n} \in \mathscr{C}_{\lambda^{2}}$. The following convergence results holds.

Theorem 4.1. Let $x$ denote the unique mild solution of (1.1) as guaranteed by Theorem 3.3. Then the sequence of mild solutions of (4.1) converges to $x$ in $X_{T, 2}$ as $n \rightarrow \infty$.

Proof. Observe that

$$
\begin{aligned}
& E\left\|x_{n}(t+\theta)-x(t+\theta)\right\|^{2} \\
\leq & 8\left[\|S(t+\theta)(n R(n ; A)-I) \phi(0)\|^{2}\right. \\
& +M_{s}^{2} T \int_{0}^{t+\theta} E\left\|n R(n ; A) f_{1}\left(s,\left(x_{n}\right)_{s}, \mu_{n}(s)\right)-f_{1}\left(s, x_{s}, \mu(s)\right)\right\|^{2} d s \\
& +\left(M_{s} M_{\mathcal{K}} T\right)^{2} \\
& \times \int_{0}^{t+\theta} \int_{0}^{s} E\left\|n R(n ; A) f_{2}\left(\tau,\left(x_{n}\right)_{\tau}, \mu_{n}(\tau)\right)-f_{2}\left(\tau, x_{\tau}, \mu(\tau)\right)\right\|^{2} d s d \tau \\
& \left.+E\left\|\int_{0}^{t+\theta} S(t+\theta-s)[n R(n ; A)-I] g(s) d B^{\mathcal{H}}(s)\right\|^{2}\right] \\
= & 8\left[\|S(t+\theta)(n R(n ; A)-I) \phi(0)\|^{2}+\sum_{i=4}^{6} I_{i}(t+\theta)\right] .
\end{aligned}
$$

Standard computations lead to

$$
\begin{aligned}
I_{4}(t+\theta) \leq & M_{s}^{2} T \int_{0}^{t+\theta}\left[E\left\|(n R(n ; A)-I) f_{1}\left(s,\left(x_{n}\right)_{s}, \mu_{n}(s)\right)\right\|^{2}\right. \\
& \left.+2 M_{f_{1}}^{2} E\left(\left\|\left(x_{n}\right)_{s}-x_{s}\right\|_{C_{r}}^{2}\right)+\rho^{2}\left(\mu_{n}(s), \mu(s)\right)\right] d s,
\end{aligned}
$$


for all $0 \leq t \leq T$. Furthermore, the triangle inequality and (A2) imply

$$
\begin{aligned}
& \int_{0}^{t+\theta} E\left\|(n R(n ; A)-I) f_{1}\left(s,\left(x_{n}\right)_{s}, \mu_{n}(s)\right)\right\|^{2} d s \\
\leq & 2 \int_{0}^{t}\|n R(n ; A)-I\|_{\mathfrak{B} \mathfrak{L}(H)}^{2}\left[2 M _ { f _ { 1 } } ^ { 2 } \left(E\left\|\left(x_{n}\right)_{s}-x_{s}\right\|_{C_{r}}^{2}\right.\right. \\
& \left.\left.+\rho^{2}\left(\mu_{n}(s), \mu(s)\right)+E\left(\left\|f_{1}\left(s, x_{s}, \mu(s)\right)\right\|^{2}\right)\right)\right] d s, \quad 0 \leq t \leq T .
\end{aligned}
$$

The boundedness $E\left(\left\|f_{1}\left(s, x_{s}, \mu(s)\right)\right\|^{2}\right)$ independent of $n$, together with the strong convergence of $n R(n ; A)$ to the zero operator, allows us to infer that the right-side of (4.4) goes to 0 as $n \rightarrow \infty$. Similar computations lead to

$$
\begin{aligned}
& I_{5}(t+\theta) \\
\leq & 2\left(M_{s} M_{\mathcal{K}} T\right)^{2} \int_{0}^{t+\theta} \int_{0}^{s}\left[E\left\|(n R(n ; A)-I) f_{2}\left(\tau,\left(x_{n}\right)_{\tau}, \mu_{n}(\tau)\right)\right\|^{2}\right. \\
& \left.+2 M_{f_{2}}^{2} E\left(\left\|\left(x_{n}\right)_{\tau}-x_{\tau}\right\|_{C_{r}}^{2}\right)+\rho^{2}\left(\mu_{n}(\tau), \mu(\tau)\right)\right] d s d \tau,
\end{aligned}
$$

for all $0 \leq t \leq T$, and using Lemma 3.2(i) yields

$$
I_{6}(t+\theta) \leq\left(\sup _{0 \leq t \leq T} C_{t} \sum_{j=1}^{\infty} v_{j}\right)\|n R(n ; A)-I\|_{\mathfrak{B} \mathfrak{L}(H)}^{2} .
$$

Using (4.3)-(4.6) in (4.2) gives rise to an inequality of the form

$$
\begin{aligned}
& E\left\|\left(x_{n}\right)_{t}-x_{t}\right\|_{C_{r}}^{2} \\
\leq & \beta_{n}^{1}+\beta^{2} \int_{0}^{t} E\left\|\left(x_{n}\right)_{s}-x_{s}\right\|_{C_{r}}^{2} d s+\beta^{3} \int_{0}^{t} \int_{0}^{s} E\left\|\left(x_{n}\right)_{\tau}-x_{\tau}\right\|^{2} d \tau d s,
\end{aligned}
$$

where $\beta_{n}^{1}$ is a constant multiple of $\|n R(n ; A)-I\|_{\mathfrak{B} \mathfrak{L}(H)}$ and $\beta^{i}(i=2,3)$ are independent of $n$, yet depend explicitly on $M_{f_{i}}, M_{\mathcal{K}}, M_{S}$ and $T$ 
$(i=1,2)$. By Lemma 2.3 and taking the supremum of (4.7) over $[0, T]$ yields

$\left\|x_{n}-x\right\|_{X_{T, 2}} \leq \beta_{n}^{1}\left[1+T \beta^{2} \exp \left(\beta^{2} T\right)\left(1+T \beta^{3} \exp \left(\beta^{3} T\right)\right)\right]$, for all $n \geq 1$.

Since the right-side of (4.8) goes to 0 as $n \rightarrow \infty$, we have the conclusion.

Corollary 4.2. The sequence of probability laws $\mu_{n}$ corresponding to the mild solutions $x_{n}$ of (4.1) converges in $\mathscr{C}_{\lambda^{2}}$ to the probability law $\mu$ corresponding to the mild solution $x$ of (1.1) as $n \rightarrow \infty$.

Proof. This follows from the fact that

$$
\begin{aligned}
D_{T}^{2}\left(\mu_{n}, \mu\right) & =\sup _{0 \leq t \leq T} \rho^{2}\left(\mu_{n}(t), \mu(t)\right) \leq \sup _{0 \leq t \leq T} E\left\|x_{n}(t)-x(t)\right\|^{2} \\
& \leq\left\|x_{n}-x\right\|_{X_{T, 2}} \rightarrow 0 \text { as } n \rightarrow \infty
\end{aligned}
$$

Remark 4.3. We observe for later purposes that Corollary 4.2 implies that

$$
\sup _{n \in \mathbb{N}} \sup _{-r \leq s \leq T}\left\|\mu_{n}(s)\right\|_{\lambda^{2}}^{2}<\infty
$$

Theorem 4.4. In addition to the assumptions of Theorem 4.1, if $\phi \in$ $L^{4}\left(\Omega, C_{r}\right)$, then

$$
P_{x_{n}} \stackrel{w}{\rightarrow} P_{x} \text { as } n \rightarrow \infty
$$

Proof. We will show that $\left\{P_{x_{n}}\right\}_{n=1}^{\infty}$ is relatively compact by using the Arzelà-Ascoli. Throughout the proof, $C_{i}$ will denote a suitable positive constant. First, we show that $\left\{x_{n}\right\}$ is uniformly bounded in $X_{T, 2}$, that is,

$$
\sup _{n \in \mathbb{N}} \sup _{0 \leq t \leq T} E\left\|\left(x_{n}\right)_{t}\right\|_{C_{r}}^{2}<\infty .
$$


For $t \in(0, T]$, the mild solution of $x_{n}$ is given by the variation of parameters formula

$$
\begin{aligned}
x_{n}(t)= & S(t) n R(n ; A) \phi(0)+\int_{0}^{t} S(t-s) n R(n ; A) f_{1}\left(s,\left(x_{n}\right)_{s}, \mu_{n}(s)\right) d s \\
& +\int_{0}^{t} S(t-s) \int_{0}^{s} \mathcal{K}(s, \tau) n R(n ; A) f_{2}\left(\tau,\left(x_{n}\right)_{\tau}, \mu_{n}(\tau)\right) d \tau d s \\
& +\int_{0}^{t} S(t-s) n R(n ; A) g(s) d B^{\mathcal{H}}(s) \\
= & S(t) n R(n ; A) \phi(0)+\sum_{i=7}^{9} I_{i}(t)
\end{aligned}
$$

and when $-r \leq t \leq 0$, the mild solution is given by $x_{n}(t)=n R(n ; A) \phi(t)$.

Let $t \in[0, T]$. For all $-r \leq \theta \leq 0$ for which $t+\theta \leq 0$, we have

$$
\begin{aligned}
& \sup _{n \in \mathbb{N}} \sup _{0 \leq t \leq T}\left\{E\left\|x_{n}(t+\theta)\right\|^{2}:-r \leq t+\theta \leq 0\right\} \\
= & \sup _{n \in \mathbb{N}} \sup _{0 \leq t \leq T}\left\{E\|n R(n ; A) \phi(t+\theta)\|^{2}:-r \leq t+\theta \leq 0\right\}<\infty,
\end{aligned}
$$

since $n R(n ; A)$ is contractive, for all $n$. Next, let $-r \leq \theta \leq 0$ be such that $0 \leq t+\theta \leq T$. We consider each of the four terms in (4.11) separately. First,

$$
E\|S(t+\theta) n R(n ; A) \phi(0)\|_{C_{r}}^{2} \leq M_{S}^{2}\|\phi(0)\|_{C_{r}}^{2} .
$$

Standard computations, taking into account (A1), (A2), and Remark 4.3, lead to

$$
E\left\|I_{7}(t+\theta)\right\|_{C_{r}}^{2} \leq 2 T\left(M_{S} M_{f_{1}}\right)^{2}\left[T C_{1}+\int_{0}^{t+\theta} E\left\|\left(x_{n}\right)_{s}\right\|_{C_{r}}^{2} d s\right]
$$

for some positive $C_{1}$ independent of $n$. Similarly, there exists $C_{2}>0$ independent of $n$ such that 


$$
E\left\|I_{8}(t+\theta)\right\|_{C_{r}}^{2} \leq 2\left(T M_{S} M_{\mathcal{K}} M_{f_{1}}\right)^{2}\left[T^{2} C_{2}+\int_{0}^{t+\theta} \int_{0}^{s} E\left\|\left(x_{n}\right)_{\tau}\right\|_{C_{r}}^{2} d s d \tau\right] .
$$

Using Lemma 3.2(i) and the contractivity of $n R(n ; A)$, there exists a positive constant $C_{3}$ independent of $n$ such that

$$
E\left\|I_{9}(t+\theta)\right\|^{2} \leq C_{3} .
$$

Combining (4.13)-(4.16) with (4.11), there exist positive constants $C_{4}$, $C_{5}, C_{6}$ independent of $n$ such that

$$
E\left\|\left(x_{n}\right)_{t}\right\|_{C_{r}}^{2} \leq C_{4}+C_{5} \int_{0}^{t} E\left\|\left(x_{n}\right)_{s}\right\|_{C_{r}}^{2} d s+C_{6} \int_{0}^{t} \int_{0}^{s} E\left\|\left(x_{n}\right)_{\tau}\right\|_{C_{r}}^{2} d \tau d s,
$$

which by Lemma 2.3 shows the uniform boundedness of $\left\{x_{n}\right\}$ in $X_{T, 2}$.

Next, we establish equicontinuity by showing for every $n \in \mathbb{N}$ and $-r \leq$ $s \leq t \leq T$ as $t-s \rightarrow 0$, we have

$$
E\left\|x_{n}(t)-x_{n}(s)\right\|^{4} \rightarrow 0 \text {, independent of } n \text {. }
$$

If $-r \leq s \leq t \leq 0$, note that

$$
E\left\|x_{n}(t)-x_{n}(s)\right\|^{4}=E\|n R(n ; A)[\phi(t)-\phi(s)]\|^{4} \rightarrow 0
$$

independent of $n$, since $\phi \in L^{4}\left(\Omega, C_{r}\right)$ and $n R(n ; A)$ is contractive, for all $n$. Now consider $0 \leq s \leq t \leq T$. Since $\{S(t): t \leq 0\}$ is a semigroup,

$$
\begin{aligned}
E\|(S(t)-S(s)) n R(n ; A) \phi(0)\|^{4} & \leq E\left(\int_{S}^{t} S(\tau) n A R(n ; A) \phi(0)\right) \\
& \leq M_{S}^{4} M_{A}^{4} E\|\phi(0)\|^{4}(t-s)^{4}
\end{aligned}
$$


where $M_{A}=\|n A R(n ; A)\|_{\mathfrak{B} \mathfrak{L}(H)}$. Also,

$$
\begin{aligned}
& E\left\|I_{7}(t)-I_{7}(s)\right\|^{4} \\
& \leq E\left(\int_{0}^{s}\left\|[S(t-\tau)-S(s-\tau)] n R(n ; A) f_{1}\left(\tau,\left(x_{n}\right)_{\tau}, \mu_{n}(\tau)\right)\right\| d \tau\right. \\
& \left.\quad+\int_{S}^{t}\left\|S(t-\tau) n R(n ; A) f_{1}\left(\tau,\left(x_{n}\right)_{\tau}, \mu_{n}(\tau)\right)\right\| d \tau\right)^{4} \\
& \leq E\left(\int_{0}^{s} \int_{s-\tau}^{t-\tau}\left\|S(u) n A R(n ; A) f_{1}\left(\tau,\left(x_{n}\right)_{\tau}, \mu_{n}(\tau)\right)\right\| d u d \tau\right. \\
& \left.\quad+M_{S} \overline{M_{f_{1}}}\left[1+\|x\|_{X_{T, 2}}+\sup _{0 \leq t \leq T}\left\|\mu_{n}(t)\right\|_{\lambda^{2}}\right](t-s)\right)^{4} \\
& \leq E\left(M_{S} M_{A} T \overline{M_{f_{1}}}\left[1+\|x\|_{X_{T, 2}}+\sup _{0 \leq t \leq T}\left\|\mu_{n}(t)\right\|_{\lambda^{2}}\right](t-s)\right. \\
& \left.\quad+M_{S} \overline{M_{f_{1}}}\left[1+\|x\|_{X_{T, 2}}+\sup _{0 \leq t \leq T}\left\|\mu_{n}(t)\right\|_{\lambda^{2}}\right](t-s)\right)^{4} \\
& =C_{7}(t-s)^{4},
\end{aligned}
$$

where $C_{7}$ is independent of $n$ by Remark 4.3. Similarly,

$$
E\left\|I_{8}(t)-I_{8}(s)\right\|^{4} \leq C_{8}(t-s)^{4} .
$$

Clearly, (4.17)-(4.18) tends to zero as $|t-s| \rightarrow 0$. It remains to show

$$
E\left\|I_{9}(t)-I_{9}(s)\right\|^{4} \rightarrow 0
$$

as $|t-s| \rightarrow 0$. Observe that

$$
\begin{aligned}
& E\left\|I_{9}(t)-I_{9}(s)\right\|^{4} \\
\leq & 4\left(E\left\|\int_{0}^{s}[S(t-\tau)-S(s-\tau)] n R(n ; A) g(\tau) d B^{\mathcal{H}}(\tau)\right\|^{4}\right.
\end{aligned}
$$




$$
\left.+E\left\|\int_{S}^{t} S(t-t) n R(n ; A) g(\tau) d B^{\mathcal{H}}(\tau)\right\|^{4}\right) .
$$

Since $S(t) \in \mathfrak{B L}(H)$, for all $t \geq 0$ and $n R(n ; A)$ is contractive, the stochastic integrals in (4.20) are Gaussian processes. Therefore, we can use the moment formula for a Gaussian process along with the estimates established in Theorem 3.3 (see (3.13), (3.14), and Lemma 3.2) to conclude that the right-side of (4.20) goes to zero as $|t-s| \rightarrow 0$. Thus, the estimates (4.17)-(4.20) then yield the equicontinuity of $\left\{x_{n}\right\}$. Therefore, we conclude the family $\left\{P_{x_{n}}\right\}_{n=1}^{\infty}$ is relatively compact by Arzelà-Ascoli, and therefore tight. Hence, by Proposition 2.5, the finite-dimensional joint distribution of $P_{x_{n}}$ converges weakly to $P_{x}$, and so by Theorem 2.6, $P_{x_{n}} \stackrel{w}{\rightarrow} P_{x}$.

\section{Examples}

Example 5.1. Let $\mathscr{D}$ be a bounded domain in $\mathbb{R}^{N}$ with smooth boundary $\partial \mathscr{D}$. Consider the following initial-boundary value problem:

$$
\left\{\begin{aligned}
\partial x(t, z)= & {\left[\Delta_{z} x(t, z)+F_{1}(t, z, x(t-r, z))\right.} \\
& \left.+\int_{L^{2}(\mathscr{D})} F_{2}(t, z, y) \mu(t, z)(d y)\right] \partial t, \\
& +g(t, z) d B^{\mathcal{H}}(t) \text { a.e. on }(0, T) \times \mathscr{D}, \\
x(t, z)= & 0 \text { a.e. on }(0, T) \times \mathscr{D}, \\
x(t, z)= & \xi(t, z) \text { a.e. on }-r \leq t \leq 0,
\end{aligned}\right.
$$

where $x:[0, T] \times \mathscr{D} \rightarrow \mathbb{R}, \quad F_{1}:[0, T] \times \mathscr{D} \times \mathbb{R} \rightarrow \mathbb{R}, \quad F_{2}:[0, T] \times \mathscr{D} \times L^{2}(\mathscr{D})$ $\rightarrow L^{2}(\mathscr{D}), \mu(t, \cdot) \in \wp_{\lambda^{2}}\left(L^{2}(\mathscr{D}), g:[0, T] \times \mathscr{D} \rightarrow \mathfrak{L}\left(\mathbb{R}, L^{2}(\mathscr{D})\right)\right.$, and $\left\{B^{\mathcal{H}}(t)\right.$ $: 0 \leq t \leq T\}$ is a real $\mathrm{fBm}$. We impose the following conditions:

(A7) $F_{1}$ satisfies the Carathéodory conditions (i.e., measurable in $(t, z)$ and continuous in the third variable) such that 
Stochastic Integrodifferential Delay Equations Driven by fBm 785

(1) $\left|F_{1}(t, y, z)\right| \leq \bar{M}_{F_{1}}[1+|z|]$, for all $0 \leq t \leq T, \quad y \in \mathscr{D}, \quad z \in \mathbb{R}$ and some $\bar{M}_{F_{1}}>0$,

(2) $\left|F_{1}\left(t, y, z_{1}\right)-F_{1}\left(t, y, z_{2}\right)\right| \leq M_{F_{1}}\left|z_{1}-z_{2}\right|$, for all $0 \leq t \leq T, y \in \mathscr{D}$, $z_{1}, z_{2} \in \mathbb{R}$ and some $M_{F_{1}}>0$;

(A8) $F_{2}$ satisfies the Carathéodory conditions and

(1) $\left\|F_{2}(t, y, z)\right\|_{L^{2}(\mathscr{D})} \leq \bar{M}_{F_{2}}\left[1+\|z\|_{L^{2}(\mathscr{D})}\right]$, for all $0 \leq t \leq T, y \in \mathscr{D}$, $z \in L^{2}(\mathscr{D})$ and some $\bar{M}_{F_{2}}>0$,

(2) $F_{2}(t, y, \cdot): L^{2}(\mathscr{D}) \rightarrow L^{2}(\mathscr{D})$ is in $\mathscr{C}$, for each $0 \leq t \leq T, y \in \mathscr{D}$;

(A9) $g:[0, T] \times \mathscr{D} \rightarrow \mathfrak{L}\left(\mathbb{R}, L^{2}(\mathscr{D})\right)$, is a bounded, strongly measurable function;

(A10) $\xi$ is an $\mathfrak{F}_{0}$-measurable random variable independent of $B^{\mathcal{H}}$ with almost surely continuous paths.

We have the following theorem.

Theorem 5.2. Assume that (A7)-(A10) hold. If $4 T M_{S}^{2} M_{f_{1}}^{2}<1$, then (5.1) has a unique mild solution $x \in X_{T, 2}$ with probability law $\{\mu(t, \cdot): 0 \leq t$ $\leq T\}$.

Proof. Let $H=L^{2}(\mathscr{D})$ and $K=\mathbb{R}^{N}$. Define the operator

$$
A x(t, \cdot)=\Delta_{z} x(t, \cdot), \quad x \in H^{2}(\mathscr{D}) \cap H_{0}^{1}(\mathscr{D}) .
$$

It is known that $A$ generates a strongly continuous semigroup $\{S(t): t \geq 0\}$ on $L^{2}(\mathscr{D})$ (see [30]), so that (A1) is satisfied. Define the maps $f_{1}:[0, T] \times$ $H \times \wp_{\lambda^{2}}(H) \rightarrow H, g:[0, T] \rightarrow \mathfrak{L}(K, H)$ and $\phi:[0, T] \times \mathscr{D} \times \mathbb{R}$, respectively, by 
$f_{1}\left(t, x_{t}, \mu(t)\right)(z)=F_{1}(t, z, x(t-r, z))+\int_{L^{2}(\mathscr{D})} F_{2}(t, z, y) \mu(t, z)(d y)$,

$g(t)(z)=g(t, z)$,

$\phi(t)(z)=\xi(t, z)$.

Further, identifying $K_{1}=f_{2}=0$, we see that (5.1) can be written in the abstract form of (1.1). Clearly, (A3)-(A6) are satisfied. We now show that $f_{1}$ (given by (5.3)) satisfies (A2). To this end, observe that from (A7)(1),

$$
\begin{aligned}
\left\|F_{1}\left(t, \cdot, x_{t}(\theta, \cdot)\right)\right\|_{L^{2}(\mathscr{D})} & \leq \bar{M}_{F_{1}}\left[\int_{\mathscr{D}}\left[1+\left|x_{t}(\theta, z)\right|\right] d z\right]^{\frac{1}{2}} \\
& \leq 2 \bar{M}_{F_{1}}\left[m\left(\mathscr{D}+\left\|x_{t}(\theta, \cdot)\right\|_{L^{2}(\mathscr{D})}^{2}\right]^{\frac{1}{2}}\right. \\
& \leq 2 \bar{M}_{F_{1}}\left[\sqrt{m(\mathscr{D})}+\left\|x_{t}\right\|_{C_{r}}^{2}\right] \\
& \leq M_{F_{1}}^{*}\left[1+\left\|x_{t}\right\|_{C_{r}}^{2}\right],
\end{aligned}
$$

for all $0 \leq t \leq T$ and $x_{t} \in C_{r}$, where

$$
M_{F_{1}}^{*}= \begin{cases}2 \bar{M}_{F_{1}} \sqrt{m(\mathscr{D})}, & \text { if } m(\mathscr{D})>1, \\ \sqrt{m(\mathscr{D})}, & \text { if } m(\mathscr{D}) \leq 1\end{cases}
$$

(Here, $m$ denotes the Lebesgue measure in $\mathbb{R}^{N}$.) Also, from (A7)(2), we obtain that

$$
\begin{aligned}
\left\|F_{1}\left(t, \cdot, x_{t}(\theta, \cdot)\right)-F_{1}\left(t, \cdot, y_{t}(\theta, \cdot)\right)\right\|_{L^{2}(\mathscr{D})} & \leq M_{F_{1}}\left[\int_{\mathscr{D}}\left|x_{t}(\theta, z)-y_{t}(\theta, z)\right|^{2}\right]^{\frac{1}{2}} \\
& =M_{F_{1}}\left\|x_{t}-y_{t}\right\|_{C_{r}}
\end{aligned}
$$

for all $0 \leq t \leq T$ and $x_{t}, y_{t} \in C_{r}$. Next, using (A8)(1) together with Hölder 
inequality, we observe that

$$
\begin{aligned}
& \left\|\int_{L^{2}(\mathscr{D})} F_{2}(t, \cdot, y) \mu(t, \cdot)(d y)\right\|_{L^{2}(\mathscr{D})} \\
= & {\left[\int_{\mathscr{D}}\left[\int_{L^{2}(\mathscr{D})} F_{2}(t, z, y) \mu(t, z)(d y)\right]^{2} d z\right]^{\frac{1}{2}} } \\
\leq & {\left[\int_{\mathscr{D}} \int_{L^{2}(\mathscr{D})}\left\|F_{2}(t, z, y)\right\|_{L^{2}(\mathscr{D})}^{2} \mu(t, z)(d y) d z\right]^{\frac{1}{2}} } \\
\leq & \bar{M}_{F_{2}}\left[\int_{\mathscr{D}} \int_{L^{2}(\mathscr{D})}\left(1+\|y\|_{L^{2}(\mathscr{D})}\right)_{L^{2}(\mathscr{D})}^{2} \mu(t, z)(d y) d z\right]^{\frac{1}{2}} \\
\leq & \bar{M}_{F_{2}} \sqrt{m(\mathscr{D})} \sqrt{\|\mu(t)\|_{\lambda^{2}}} \\
\leq & \bar{M}_{F_{2}} \sqrt{m(\mathscr{D})}\left(1+\sqrt{\left.\|\mu(t)\|_{\lambda^{2}}\right)}, \forall 0 \leq t \leq T, \mu \in \wp \lambda^{2}(H) .\right.
\end{aligned}
$$

Also, invoking (A8)(2) enables us to see that for all $\mu, v \in \wp_{\lambda^{2}}(H)$,

$$
\begin{aligned}
& \left\|\int_{L^{2}(\mathscr{D})} F_{2}(t, \cdot, y) \mu(t, \cdot)(d y)-\int_{L^{2}(\mathscr{D})} F_{2}(t, \cdot, y) v(t, \cdot)(d y)\right\|_{L^{2}(\mathscr{D})} \\
= & \int_{L^{2}(\mathscr{D})} F_{2}(t, \cdot y)(\mu(t, \cdot)-v(t, \cdot))(d y) \\
\leq & \|\rho(\mu(t), v(t))\|_{L^{2}(\mathscr{D})} \\
\leq & \sqrt{m(\mathscr{D})} \rho(\mu(t), v(t)), \forall 0 \leq t \leq T .
\end{aligned}
$$

Combining (5.6) and (5.9), we see that $f_{1}$ satisfies (A2)(a) with

$$
\bar{M}_{f_{1}}=2 \max \left\{\bar{M}_{F_{2}} \sqrt{m(\mathscr{D})}, M^{*} F_{1}\right\},
$$

and combining (5.7) and (5.10), we see that $f_{1}$ satisfies (A2)(b) with

$$
\bar{M}_{f_{1}}=\max \left\{M_{F_{1}}, \sqrt{m(\mathscr{D})}\right\}
$$


Thus, we can invoke Theorem 3.3 to conclude that (5.1) has a unique mild solution $x \in X_{T, 2}$ with probability law $\{\mu(t): 0 \leq t \leq T\}$.

Example 5.3. Consider the following initial-boundary value problem of Sobolev type:

$$
\left\{\begin{aligned}
& \partial\left(x(t, z)-\alpha x_{z z}(t, z)\right) \partial t \\
= & \left(\beta x_{z z}(t, z)+F_{1}(t, z, x(t-r, z))+\int_{L^{2}(-1,1)} F_{2}(t, z, y) \mu(t, z)(d y)\right) \partial t \\
& +g(t, z) d B^{\mathcal{H}}(t) \text { a.e. on }(0, T) \times(-1,1), \\
& x(t, z)=0 \text { a.e. on }(0, T) \times(-1,1), \\
& x(t, z)=\xi(t, z) \text { a.e. on }-r \leq t \leq 0,
\end{aligned}\right.
$$

where $\quad x:[0, T] \times(-1,1) \rightarrow \mathbb{R}, \quad F_{1}:[0, T] \times(-1,1) \times \mathbb{R} \rightarrow \mathbb{R} \quad$ and $\quad F_{2}:$ $[0, T] \times(-1,1) \times L^{2}(-1,1) \rightarrow L^{2}(-1,1)$ satisfy $(\mathrm{A} 7)$ and $(\mathrm{A} 8), \mu(t, \cdot) \in$ $\wp_{\lambda^{2}}\left(L^{2}(-1,1)\right), g:[0, T] \times(-1,1) \rightarrow \mathfrak{L}\left(\mathbb{R}, L^{2}(-1,1)\right)$ is a bounded, strongly measurable mapping, $\left\{B^{\mathcal{H}}(t): 0 \leq t \leq T\right\}$ is a real $\mathrm{fBm}$, and $\alpha, \beta$ are real, positive constants. We have the following theorem.

Theorem 5.4. Under the above assumptions, (5.11) has a unique mild solution $x \in X_{T, 2}$, provided that $4 T M_{f_{1}}^{2}<1$.

Proof. Define $H=L^{2}(-1,1), K=\mathbb{R}$, and define the operators $A: D(A)$ $\subset H \rightarrow H$ and $B: D(B) \subset H \rightarrow H$, respectively, by

$$
A x(t, \cdot)=-\beta x_{z z}(t, \cdot), \quad B x(t, \cdot)=x(t, \cdot)-\alpha x_{z z}(t, \cdot)
$$

with domains

$$
\begin{aligned}
D(A) & =D(B) \\
& =\left\{x \in L^{2}(-1,1): x, x_{z}\right. \text { are absolutely continuous, }
\end{aligned}
$$




$$
\left.x_{z z} \in L^{2}(-1,1), x(-1)=x(1)=0\right\} .
$$

Define $f_{1}, K$, and $\phi$ as in the previous example (with $L^{2}(-1,1)$ in place of $\left.L^{2}(\mathscr{D})\right)$. Then $(5.11)$ can be written in the abstract form

$$
\begin{aligned}
& d v(t)=-A B^{-1} v(t) d t+f_{1}\left(t, B^{-1} v_{t}, \mu(t)\right) d t+g(t) B^{\mathcal{H}}(t), t \in(0, T), \\
& v(0)=B \phi(t),-r \leq t \leq 0 .
\end{aligned}
$$

It is known that $B$ is a bijective operator possessing a continuous inverse and that $-A B^{-1}$ is a bounded linear operator on $L^{2}(-1,1)$ which generates a strongly continuous semigroup $\{S(t): t \geq 0\}$ on $L^{2}(-1,1)$ satisfying (A1) with $M_{s}=1$ (see [30]). Furthermore, $f_{1}$ can be shown to satisfy (A2) as in Example 5.1. Consequently, we can invoke Theorem 3.3 (assuming $\left.4 T M_{f_{1}}^{2}<1\right)$ to conclude (5.14) has a unique mild solution $v \in X_{T, 2}$. Consequently, $x=B^{-1} v$ is the corresponding mild solution of (5.11).

\section{Numerical Experiments}

Consider the interacting stochastic particle system

$$
\left\{\begin{aligned}
d X^{i}(t)= & {\left[A X^{i}(t)+F_{1}^{1}\left(t, X_{t-r}^{i}\right)+\frac{1}{M} \sum_{j=1}^{M} F_{2}^{1}\left(t, X^{j}(t)\right)\right.} \\
& \left.+\int_{0}^{t} \mathcal{K}(t, s)\left[F_{1}^{2}\left(t, X_{t-r}^{i}\right)+\frac{1}{M} \sum_{j=1}^{M} F_{2}^{2}\left(t, X^{j}(t)\right)\right] d s\right] d t \\
& +g(t) d B^{\mathcal{H}, i}(t), \quad 0 \leq t \leq T, \\
X^{i}(t)= & \phi(t), \quad-r \leq t \leq 0
\end{aligned}\right.
$$

for $i=1,2, \ldots, M$ and where $B^{\mathcal{H}, 1}(t), \ldots, B^{\mathcal{H}, M}(t)$ are independent fBms. Following the approaches given in $[32,31,35,6,7]$, we have made the ansatz that the empirical measure, 


$$
\mu_{M}(t)=\frac{1}{M} \sum_{j=1}^{M} \delta_{X^{j}(t)}
$$

approximates $\mu(t)$ in order to relate (1.1) to (6.1). Furthermore, we assumed that $f_{1}$ and $f_{2}$ from (1.1) are of the form

$$
\begin{aligned}
f_{i}\left(t, x_{t}, \mu(t)\right)(z)= & F_{1}^{i}\left(t, x_{t}\right)(z)+\int_{L^{2}(\mathscr{D})} F_{2}^{i}(t, y)(z) \mu(t)(z)(d y) \\
= & F_{1}^{i}(t, z, x(t-r, z)) \\
& +\int_{L^{2}(\mathscr{D})} F_{2}^{i}(t, z, y) \mu(t, z)(d y), i=1,2
\end{aligned}
$$

where $F_{1}^{i}, F_{2}^{i}$ are such that $f_{i}$ satisfies (A2).

We are not claiming solutions to (6.1) converge to solutions of (1.1) as $M$ tends to infinity. This property is commonly referred to as propagation of chaos and originates with Kac's Markovian models of gas dynamics [17]. Propagation of chaos has been studied for systems similar to (1.1) [32, 31, $35,6,7]$, but those results applied to stochastic differential equations or equations driven by Brownian motion. To the authors' knowledge, there is no analogous result for an SPDE similar to (1.1). Since we are not providing such a result to relate (1.1) to (6.1), the examples that follow are meant only to illustrate solution trajectories of (6.1).

For each $i \in\{1, \ldots, M\}$, the mild solutions to (6.1) are given by

$$
\begin{aligned}
X^{i}(t)= & S(t) \phi(0)+\int_{0}^{t} S(t-\tau)\left[F_{1}^{1}\left(\tau, X_{\tau-r}^{i}\right)+\frac{1}{M} \sum_{j=1}^{M} F_{2}^{1}\left(\tau, X^{j}(\tau)\right)\right] d \tau \\
& +\int_{0}^{t} S(t-\tau) \int_{0}^{\tau} \mathcal{K}(\tau, s)\left[F_{1}^{2}\left(s, X_{s-r}^{i}\right)+\frac{1}{M} \sum_{j=1}^{M} F_{2}^{2}\left(s, X^{j}(s)\right)\right] d s d \tau
\end{aligned}
$$




$$
\begin{gathered}
\quad+\int_{0}^{t} S(t-\tau) g(\tau) d B^{\mathcal{H}, i}(\tau), \quad 0 \leq t \leq T, \\
X^{i}(t)=\phi(t), \quad-r \leq t \leq 0 .
\end{gathered}
$$

In practice, the $X^{i}(t)^{\prime}$ s cannot be computed explicitly and will be approximated by a discrete-time process $\bar{X}_{k \Delta t}^{i}$, where $\Delta t$ is a discretization step of the time interval $[0, T]$. To this end, let $\Delta t>0$ and $c_{T}, c_{r} \in \mathbb{N}$ be such that $T=c_{T} \Delta t$ and $r=c_{r} \Delta t$. The discrete times are denoted by $t_{k}=k \Delta t$, with $k \in\left\{1,2, \ldots, c_{T}\right\}$. Evaluating (6.3) at the discrete time $t_{k+1}$ and using the semigroup property yields

$$
\begin{aligned}
& X^{i}\left(t_{k+1}\right)= S(\Delta t) X^{i}\left(t_{k}\right) \\
&+\int_{t_{k}}^{t_{k+1}} S\left(t_{k+1}-\tau\right)\left\{\left[F_{1}^{1}\left(\tau, X_{\tau-r}^{i}\right)+\frac{1}{M} \sum_{j=1}^{M} F_{2}^{1}\left(\tau, X^{j}(\tau)\right)\right.\right. \\
&\left.+\int_{0}^{\tau} \mathcal{K}(\tau, s)\left[F_{1}^{2}\left(s, X_{s-r}^{i}\right)+\frac{1}{M} \sum_{j=1}^{M} F_{2}^{2}\left(s, X^{j}(s)\right)\right] d s\right] d \tau \\
&\left.+g(\tau) d B^{\mathcal{H}, i}(\tau)\right\}, \\
& X^{i}(t)=\phi(t), \quad-r \leq t \leq 0,
\end{aligned}
$$

for each $i \in\{1, \ldots, M\}$. To obtain a viable simulation procedure, we use an Euler scheme on (6.4) to produce the following algorithm for the discretetime process $\bar{X}_{t_{k+1}}^{i}$ :

$$
\bar{X}_{t_{k+1}}^{i}=S(\Delta t)\left\{\bar{X}_{t_{k}}^{i}+\left[F_{1}^{1}\left(k \Delta t, \bar{X}_{t_{k}-r}^{i}\right)+\frac{1}{M} \sum_{j=1}^{M} F_{2}^{1}\left(t_{k}, \bar{X}^{j}\left(t_{k}\right)\right)\right] \Delta t\right.
$$




$$
\begin{aligned}
& +\sum_{l=0}^{k-1} \mathcal{K}\left(t_{k}, l \Delta t\right)\left[F_{1}^{2}\left(l \Delta t, \bar{X}_{l \Delta t-r}^{i}\right)+\frac{1}{M} \sum_{j=1}^{M} F_{2}^{2}\left(l \Delta t, \bar{X}^{j}(l \Delta t)\right)\right] \Delta t \Delta t \\
& \left.+g\left(t_{k}\right)\left[B^{\mathcal{H}, i}\left(t_{k+1}\right)-B^{\mathcal{H}, i}\left(t_{k}\right)\right]\right\}, \quad k \in\left\{0,1, \ldots, c_{T}-1\right\}, \\
\bar{X}_{-l \Delta t}^{i}= & \phi(-l \Delta t), \quad l \in\left\{0, \ldots, c_{r}\right\} .
\end{aligned}
$$

Thus, we approximate $\mu_{M}\left(t_{k}\right)$ by the empirical measure

$$
\bar{\mu}_{M}\left(t_{k}\right)=\frac{1}{M} \sum_{j=1}^{M} \delta_{\bar{X}_{t_{k}}^{j}} .
$$

We present results of numerical experiments corresponding to Examples 5.1 and 5.3. Our objective is to illustrate solutions and provide numerical evidence for weak convergence of (6.5) to (6.4) with respect to decreasing the time step size. We perform the numerical simulations using MATLAB and the compute fractional Brownian motion increments, $d B^{\mathcal{H}}\left(t_{k}\right)$, using the MATLAB function wfom.m. We denote the length of the synthesized fractional Brownian motion using wftom. m by $N_{T}$.

In the next two examples, we fix $\mathcal{H}=0.6, T=1$, and $\Delta t=T /\left(N_{T}-1\right)$, where $N_{T}=100$. The various approximation errors at $T=1$ are defined as follows:

$$
\begin{aligned}
& E_{1}=\left|\mathbf{E}\left\|\bar{X}_{1}\right\|_{L^{2}(\mathscr{D})}-\mathbf{E}\|X(1)\|_{L^{2}(\mathscr{D})}\right|, \\
& E_{2}=\left\|\mathbf{E} \bar{X}_{1}-\mathbf{E} X(1)\right\|_{L^{2}(\mathscr{D})}, \\
& E_{3}=\left|\mathbf{E}\left\|\varphi\left(\bar{X}_{1}\right)\right\|_{L^{2}(\mathscr{D})}-\mathbf{E}\|\varphi(X(1))\|_{L^{2}(\mathscr{D})}\right|, \\
& E_{4}=\left\|\mathbf{E} \varphi\left(\bar{X}_{1}\right)-\mathbf{E} \varphi(X(1))\right\|_{L^{2}(\mathscr{D})},
\end{aligned}
$$

where $\varphi(u)=u^{4}$ and $\mathbf{E}$ denotes the expectation of the $M$ samples. 
Example 6.1. In Example 5.1, we considered

$$
\left\{\begin{aligned}
\partial x(t, z)= & {\left[\Delta_{z} x(t, z)+F_{1}(t, z, x(t-r, z))\right.} \\
& \left.+\int_{L^{2}(\mathscr{D})} F_{2}(t, z, y) \mu(t, z)(d y)\right] \partial t \\
& +g(t, z) d B^{H}(t) \text { a.e. on }(0, T) \times \mathscr{D}, \\
x(t, z)= & 0 \text { a.e. on }(0, T) \times \mathscr{D}, \\
x(t, z)= & \xi(t, z) \text { a.e. on }-r \leq t \leq 0
\end{aligned}\right.
$$

and used Theorem 3.3 to conclude that (6.7) has a unique solution. In this example, we illustrate approximate solutions to the corresponding interacting stochastic particle system of $(6.7)$ for $\mathscr{D}=(-1,1)$. We perform numerical experiments on two cases of (6.1) with $A, g(t)$, and $\phi(t)$ as defined in (5.2), (5.4), and (5.5), respectively. Case 1 assumes

$$
F_{1}^{1}(t, y)=e^{-|y|}, \quad F_{2}^{1}(t, y)=y, \quad F_{1}^{2}(t, y)=0=F_{2}^{2}(t, y), \quad r=10 \Delta t
$$

and

$$
\phi(t)(z)=\frac{1}{2} e^{-40(z+|t|)^{2}}, \quad g(t)[y]=e^{-t} y .
$$

Case 2 is the same as Case 1 except for $F_{1}^{2}(t, y)=e^{-|y|}$ and

$$
g(t)[y]=\cos (t) \mathbb{S}^{-1} \frac{2}{2 k+1} \mathbb{S}[y] .
$$

The sine transform on $(-1,1), \mathbb{S}$, is given by

$$
\mathbb{S}[y](k)=\int_{-1}^{1} y(z) \sin \left(\frac{k \pi}{2}(z+1)\right) d z, k \in \mathbb{Z}
$$

and $\mathbb{S}^{-1}$ denotes the its inverse.

Since it is not practical to calculate the solution to (6.1), we use the explicit Euler method defined in (6.5) to approximate the solution. We employ spectral methods to approximate $S(\Delta t)$ using $N_{z}$ interior nodes 
of $(-1,1)$. We omit the details of spectral methods and decompositions and refer an interested reader to [34]. In order to compute the errors given in (6.6), we define the exact solution as the solution to (6.5) for $N_{z}=2^{9}$, $\Delta t=\Delta t / 4$ and $M=1000$.

Figures 1 and 2 contain the results of our numerical simulation for $N_{z}=2^{7}, \quad M=100$ and decreasing time steps: $\Delta t, \Delta t / 2, \Delta t / 4$. Figures 1(a) and 2(a) illustrate the evolution in time of 10 randomly selected sample paths at $z=0$. Figures 1(b) and 2(b) contain the surface plot of the mean solution to each of the cases. Lastly, Figures 1(c) and 2(c) provide some numerical evidence for weak convergence as the size of the time step tends to zero.

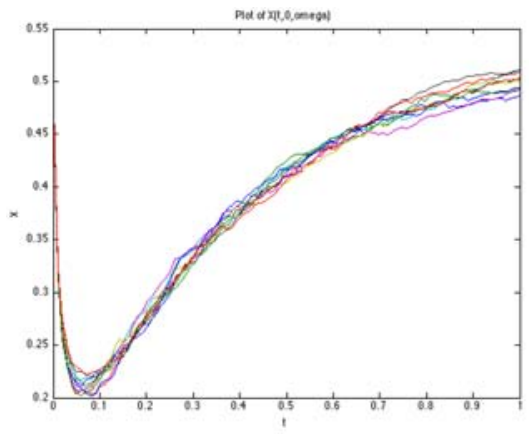

(a) Sample trajectories at $z=0$

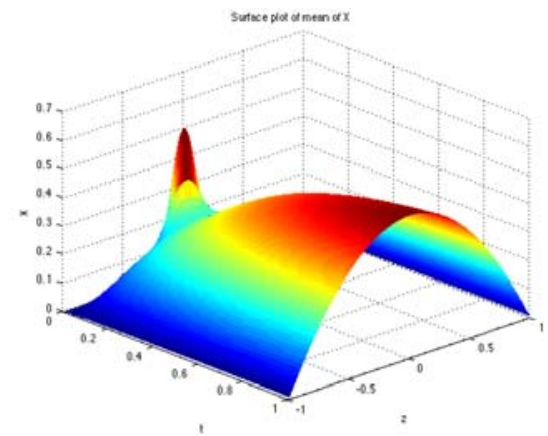

(b) Surface plot of mean solution

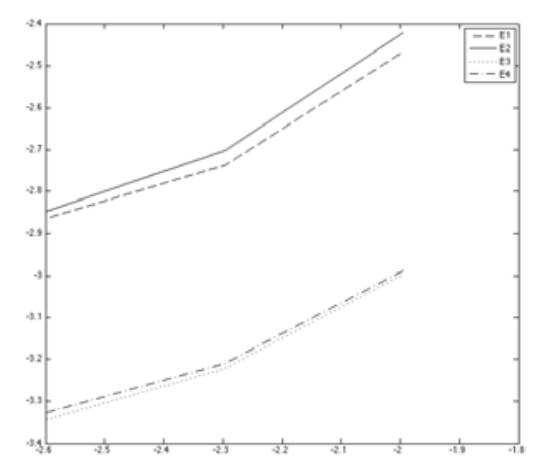

(c) $\log$ (error) vs. $\log (\Delta t)$

Figure 1. Example 6.1, Case 1. 


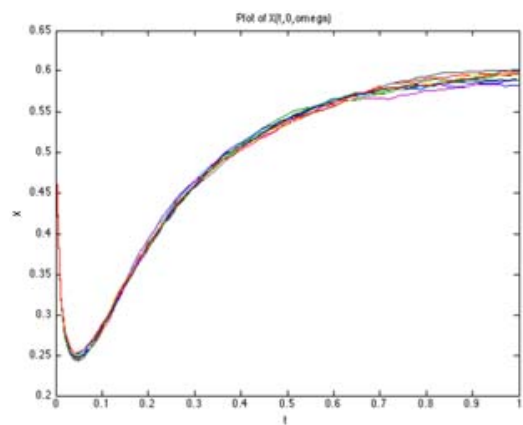

(a) Sample trajectories at $z=0$

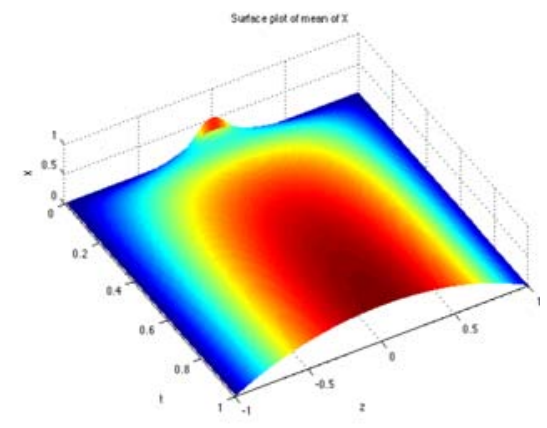

(b) Surface plot of mean solution

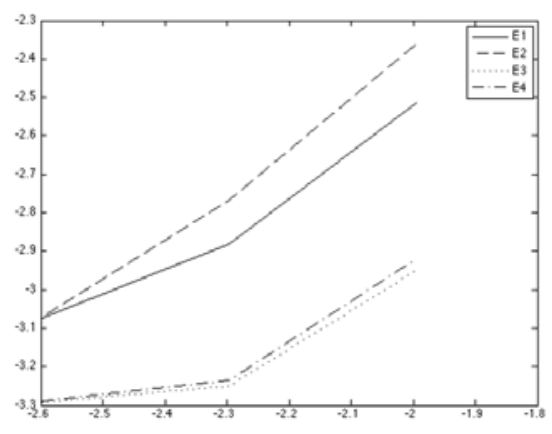

(c) $\log$ (error) vs. $\log (\Delta t)$

Figure 2. Example 6.1, Case 2.

Example 6.2. In Example 5.3, we introduced the following initialboundary value problem of Sobolev type:

$$
\left\{\begin{array}{l}
\partial\left(x(t, z)-\alpha x_{z z}(t, z)\right) \partial t \\
=\left(\beta x_{z z}(t, z)+F_{1}(t, z, x(t-r, z))\right. \\
\left.\quad+\int_{L^{2}(-1,1)} F_{2}(t, z, y) \mu(t, z)(d y)\right) \partial t \\
\quad+g(t, z) d B^{H}(t) \text { a.e. on }(0, T) \times(-1,1), \\
z(t, z)=0 \text { a.e. on }(0, T) \times(-1,1), \\
x(t, z)=\xi(t, z) \text { a.e. on }-r \leq t \leq 0 .
\end{array}\right.
$$

Theorem 3.3 was used to conclude that (6.8) has a unique solution. In this example, we illustrate solutions to the corresponding interacting stochastic particle system of (6.8). We perform numerical experiments on 
two cases of (6.1) with $A, g(t)$ and $\phi(t)$ as defined as in Theorem 5.4. Case 1 assumes

$$
F_{1}^{1}(t, y)=e^{-|y|}, \quad F_{2}^{1}(t, y)=y, \quad F_{1}^{2}(t, y)=0=F_{2}^{2}(t, y), \quad r=10 \Delta t
$$

and

$$
\phi(t)(z)=\frac{1}{2} e^{-40(z+|t|)^{2}}, \quad g(t)[y]=\cos (t) \mathbb{S}^{-1} \frac{2}{2 k+1} \mathbb{S}[y] .
$$

Case 2 is the same as Case 1 except for $F_{1}^{2}(t, y)=\cos (\pi y t)$ and

$$
\phi(t)(z)=\sin (t) \sin (\pi z) .
$$

Furthermore, both cases use $\alpha=0.25$ and $\beta=1$. As in Example 5.1, we use spectral methods to approximate $S(\Delta t)$ and define the exact solution in the same manner.

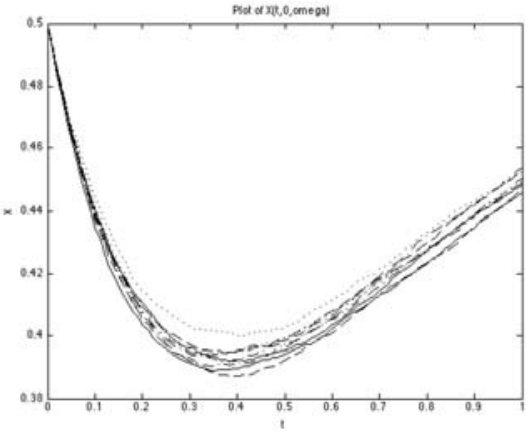

(a) Sample trajectories at $z=0$

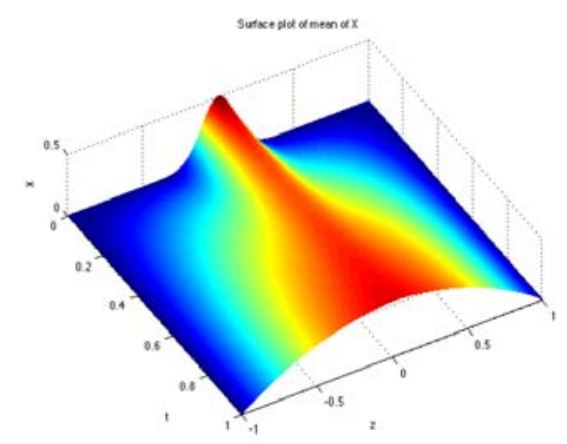

(b) Surface plot of mean solution

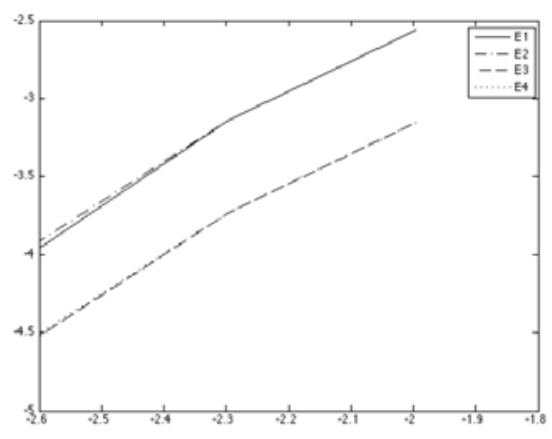

(c) $\log$ (error) vs. $\log (\Delta t)$

Figure 3. Example 6.2, Case 1. 


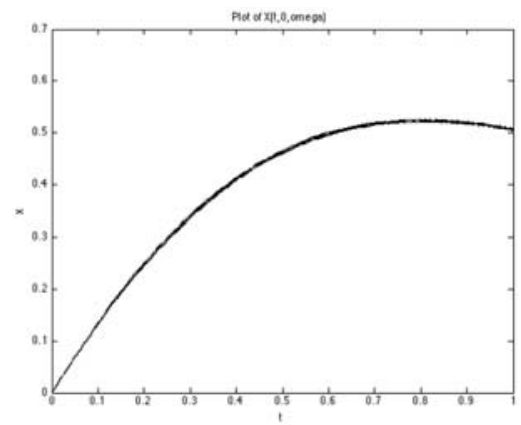

(a) Sample trajectories at $z=0$

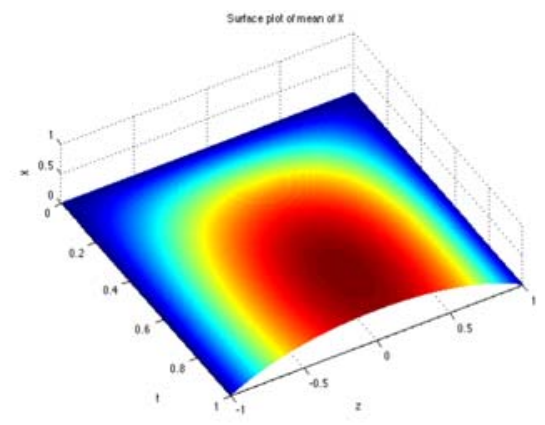

(b) Surface plot of mean solution

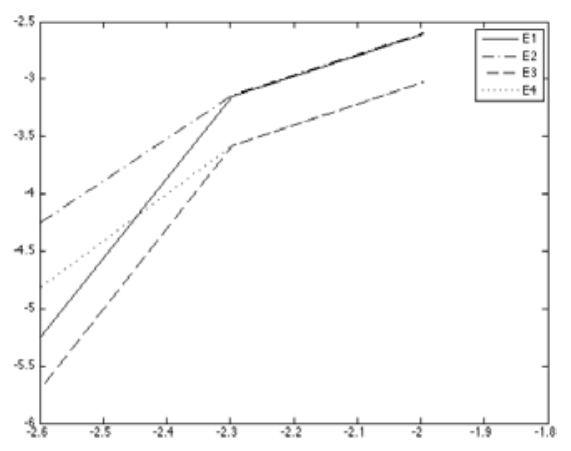

(c) $\log ($ error $)$ vs. $\log (\Delta t)$

Figure 4. Example 6.2, Case 2.

Figures 3 and 4 contain the results of our numerical simulations for $N_{z}=2^{7}, M=100$ and decreasing time steps: $\Delta t, \Delta t / 2, \Delta t / 4$. Figures 3(a) and 4(a) illustrate the evolution in time of 10 randomly selected sample paths at $z=0$. Figures 3(b) and 4(b) contain the surface plot of the mean solution to each of the cases. Lastly, Figures 3(c) and 4(c) provide some numerical evidence for weak convergence as the size of the time step tends to zero.

\section{References}

[1] N. U. Ahmed and X. Ding, A semilinear McKean-Vlasov stochastic evolution equation in Hilbert space, Stochastic Process. Appl. 60(1) (1995), 65-85.

[2] V. V. Anh and W. Grecksch, A fractional stochastic evolution equation driven by fractional Brownian motion, MCMA 9(3) (2003), 189-199. 
[3] Harald Bergström, Weak convergence of measures, Probability and Mathematical Statistics, Academic Press, Inc., 1982.

[4] A. T. Bharucha-Reid, Random integral equations, Academic Press, New YorkLondon, Mathematics in Science and Engineering, Vol. 96, 1972.

[5] Patrick Billingsley, Weak convergence of measures: applications in probability, Society for Industrial and Applied Mathematics, Philadelphia, Pa., Conference Board of the Mathematical Sciences Regional Conference Series in Applied Mathematics, No. 5, 1971.

[6] Mireille Bossy and Denis Talay, Convergence rate for the approximation of the limit law of weakly interacting particles: application to the Burgers equation, Ann. Appl. Probab. 6(3) (1996), 818-861.

[7] Mireille Bossy and Denis Talay, A stochastic particle method for the McKeanVlasov and the Burgers equation, Math. Comp. 66(217) (1997), 157-192.

[8] Heinz Brill, A semilinear Sobolev evolution equation in a Banach space, J. Differential Equations 24(3) (1977), 412-425.

[9] M. G. Crandall, S.-O. Londen and J. A. Nohel, An abstract nonlinear Volterra integrodifferential equation, J. Math. Anal. Appl. 64(3) (1978), 701-735.

[10] Giuseppe Da Prato and Jerzy Zabczyk, Stochastic equations in infinite dimensions, Vol. 44 of Encyclopedia of Mathematics and its Applications, Cambridge University Press, Cambridge, 1992.

[11] Donald A. Dawson, Critical dynamics and fluctuations for a mean-field model of cooperative behavior, J. Statist. Phys. 31(1) (1983), 29-85.

[12] Donald A. Dawson and Jürgen Gärtner, Large deviations from the McKeanVlasov limit for weakly interacting diffusions, Stochastics 20(4) (1987), 247-308.

[13] L. Decreusefond and A. S. Üstünel, Stochastic analysis of the fractional Brownian motion, Potential Anal. 10(2) (1999), 177-214.

[14] T. E. Duncan, B. Pasik-Duncan and B. Maslowski, Fractional Brownian motion and stochastic equations in Hilbert spaces, Stoch. Dyn. 2(2) (2002), 225-250.

[15] T. E. Govindan, Autonomous semilinear stochastic Volterra integro-differential equations in Hilbert spaces, Dynam. Systems Appl. 3(1) (1994), 51-74.

[16] W. Grecksch and V. V. Anh, A parabolic stochastic differential equation with fractional Brownian motion input, Statist. Probab. Lett. 41(4) (1999), 337-346. 
[17] M. Kac, Foundations of kinetic theory, Proceedings of the Third Berkeley Symposium on Mathematical Statistics and Probability, 1954-1955, Vol. III, pp. 171-197, University of California Press, Berkeley and Los Angeles, 1956.

[18] D. Kannan, Random integrodifferential equations, Probabilistic Analysis and Related Topics, Academic Press, New York, Vol. 1, 1978, pp. 87-167.

[19] D. Kannan and Albert T. Bharucha-Reid, On a stochastic integro-differential evolution equation of Volterra type, J. Integral Equations 10(1-3, suppl.) (1985), 351-379.

[20] David N. Keck and Mark A. McKibben, On a McKean-Vlasov stochastic integro-differential evolution equation of Sobolev-type, Stochastic Anal. Appl. 21(5) (2003), 1115-1139.

[21] K. Knopp, Theory and Application of Infinite Series, Vol. 197 of Dover Books on Mathematics, Dover Publications, 1990.

[22] Hiroshi Kunita, Stochastic flows and stochastic differential equations, Vol. 24 of Cambridge Studies in Advanced Mathematics, Cambridge University Press, Cambridge, 1990.

[23] James H. Lightbourne, III and Samuel M. Rankin, III, A partial functionaldifferential equation of Sobolev type, J. Math. Anal. Appl. 93(2) (1983), 328-337.

[24] S. J. Lin, Stochastic analysis of fractional Brownian motions, Stochastics Stochastics Rep. 55(1-2) (1995), 121-140.

[25] S.-O. Londen and J. A. Nohel, Nonlinear Volterra integro-differential equation occurring in heat flow, J. Integral Equations 6(1) (1984), 11-50.

[26] Bohdan Maslowski and David Nualart, Evolution equations driven by a fractional Brownian motion, J. Funct. Anal. 202(1) (2003), 277-305.

[27] Masao Nagasawa and Hiroshi Tanaka, Diffusion with interactions and collisions between coloured particles and the propagation of chaos, Probab. Theory Related Fields 74(2) (1987), 161-198.

[28] David Nualart and Aurel Răşcanu, Differential equations driven by fractional Brownian motion, Collect. Math. 53(1) (2002), 55-81.

[29] B. G. Pachpatte, Inequalities for differential and integral equations, Vol. 197 of Mathematics in Science and Engineering, Academic Press, Inc. 1998.

[30] A. Pazy, Semigroups of linear operators and applications to partial differential equations, Vol. 44 of Applied Mathematical Sciences, Springer-Verlag, New York, 1983. 
[31] Dan Stanescu, Dongjin Kim and Wojbor A. Woyczynski, Numerical study of interacting particles approximation for integro-differential equations, J. Comput. Phys. 206(2) (2005), 706-726.

[32] Denis Talay, Probabilistic numerical methods for partial differential equations: elements of analysis, Probabilistic Models for Nonlinear Partial Differential Equations (Montecatini Terme, 1995), Vol. 1627 of Lecture Notes in Math. Springer, Berlin, 1996, pp. 148-196.

[33] Takeshi Taniguchi, Almost sure exponential stability for stochastic partial functional-differential equations, Stochastic Anal. Appl. 16(5) (1998), 965-975.

[34] Lloyd N. Trefethen, Spectral methods in MATLAB, Vol. 10 of Software, Environments, and Tools, Society for Industrial and Applied Mathematics (SIAM), Philadelphia, PA, 2000.

[35] A. Yu. Veretennikov, On ergodic measures for McKean-Vlasov stochastic equations, Monte Carlo and Quasi-Monte Carlo Methods, 2004, Springer, Berlin, 2006, pp. 471-486.

[36] Jianhong $\mathrm{Wu}$, Theory and applications of partial functional-differential equations, Vol. 119 of Applied Mathematical Sciences, Springer-Verlag, New York, 1996. 